\title{
On the existence of free symmetric pairs in normal subgroups of division rings with involution
}

\author{
Pedro Russo de Oliveira
}

TESE APRESENTADA

$\mathrm{AO}$

Instituto DE MATEMÁtica E Estatística

DA

Universidade de São Paulo

PARA

OBTENÇÃO DO TÍTULO

$\mathrm{DE}$

Doutor EM CIÊNCIAS

Programa: Matemática

Orientador: Prof. Dr. Jairo Zacarias Gonçalves

Durante o desenvolvimento deste trabalho, o autor recebeu auxílio financeiro da CAPES 



\section{Sobre a existência de pares simétricos livres em subgrupos normais de anéis com divisão com involução}

Esta versão da tese contém as correções e alterações sugeridas pela Comissão Julgadora durante a defesa da versão original do trabalho, realizada em 27/08/2020. Uma cópia da versão original está disponível no Instituto de Matemática e Estatística da Universidade de São Paulo.

Comissão Julgadora:

- Prof. Dr. Jairo Zacarias Gonçalves (orientador) - IME-USP

- Prof. Dr. Vitor de Oliveira Ferreira - IME-USP

- Prof. Dr. Arnaldo Mandel - IME-USP

- Profa. Dra. Érica Zancanella Fornaroli - DMA-UEM

- Prof. Dr. Eduardo Tengan - MTM-UFC 



\section{Agradecimentos}

A Jairo, a Silvia, a Felipe e a Luís. Vocês são os verdadeiros responsáveis por este trabalho. Agradeço-lhes todo o incentivo.

Aos meus familiares.

Às minhas amigas, aos meus amigos.

À comissão julgadora.

À CAPES, que me concedeu auxílio financeiro. 



\title{
Resumo
}

\author{
OLIVEIRA, P. R. Sobre a existência de pares simétricos livres em sub- \\ grupos normais de anéis com divisão com involução. Tese (Doutorado) - \\ Instituto de Matemática e Estatística, Universidade de São Paulo, São Paulo, 2020.
}

Seja $D$ um anel com divisão não comutativo de centro $\mathbb{k}$, cuja característica seja distinta de 2 , munido de uma involução $*$. Dizemos que $*$ é de primeira espécie se fixa cada um dos elementos de $\mathbb{k}$ e que é de segunda espécie em caso contrário. Um par simétrico livre em $D$ é um subconjunto $\{x, y\}$ de elementos simétricos isto é, $x^{*}=x$ e $y^{*}=y-$ não nulos de $D$ que geram livremente um grupo livre. Seja $N$ um subgrupo normal não central do grupo multiplicativo $D^{\times}=D \backslash\{0\}$. Apresentaremos, aqui, condições suficientes para que $N$ contenha pares simétricos livres, com exceção do caso em que $D$ é uma álgebra de quatérnios e * é simplética. Especificamente, mostramos que $N$ contém pares simétricos livres quando a dimensão de $D$ sobre lk é finita, nos seguintes casos: (a) * é de primeira espécie e k é não enumerável; (b) $D$ é uma álgebra de quatérnions e * é uma involução ortogonal ou de segunda espécie; (c) * é de primeira espécie e $N$ contém uma raiz da unidade simétrica. Independentemente da dimensão de $D$ e da espécie de $*$, a mesma conclusão é válida nos casos: (d) $N$ contém uma raiz da unidade simétrica cujo polinômio minimal, no caso em que $k$ tem característica positiva, tem grau par; e (e) $N$ contém um elemento simétrico algébrico sobre k cujo polinômio minimal tem grau 2.

Palavras-chave: anéis com divisão, involução, pares simétricos livres. 



\section{Abstract}

OLIVEIRA, P. R. On the existence of free symmetric pairs in normal subgroups of division rings with involution. PhD Thesis - Institute of Mathematics and Statistics of the University of São Paulo, São Paulo, 2020.

Let $D$ be a noncommutative division ring with center $\mathbb{k}$, whose characteristic is distinct from 2 , endowed with an involution $*$ - which is said to be of the first kind, if it is $\mathbb{k}$-linear, and of the second kind, otherwise. By a free symmetric pair in $D$, one understands a subset $\{x, y\}$ of symmetric - i.e., $x^{*}=x$ and $y^{*}=y$-nonzero members of $D$ which freely generate a free group. Let $N$ be a non central normal subgroup of the multiplicative group $D^{\times}=D \backslash\{0\}$. We present sufficient conditions for the existence of free symmetric pairs in $N$, with exception of the case in which $D$ is a quaternion algebra and $*$ is symplectic. Specifically, when the dimension of $D$ over $\mathbb{k}$ is finite, we show that $N$ contains free symmetric pairs in the following cases: (a) $*$ is of the first kind and $\mathbb{k}$ is uncountable; (b) $D$ is a quaternion algebra and $*$ is an orthogonal involution or an involution of the second kind; (c) $*$ is of the first kind and $N$ contains a symmetric root of unity. Without any assumption on the dimension of $D$ or on the kind of $*$, the same conclusion holds in the cases: (d) $N$ contains a symmetric root of unity whose minimal polynomial, in case $\mathrm{k}$ has positive characteristic, has even degree; and (e) $N$ contains a symmetric element which is algebraic over $\mathrm{k}$ and whose minimal polynomial has degree 2.

Keywords: division rings, involution, free symmetric pairs.

2010 Mathematics Subject Classification. Primary: 16K20, 16K40; Secondary: 20E05, $12 \mathrm{E} 15$. 



\section{Contents}

List of symbols $\quad$ xi

Introduction xiii

\begin{tabular}{lll}
\hline 1 & Preliminaries & 1
\end{tabular}

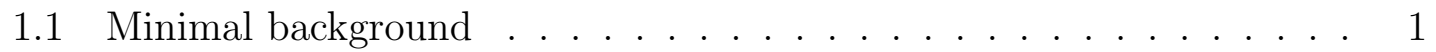

1.2 Extension of the evaluation map . . . . . . . . . . . . . . . 2

1.3 Involutions . . . . . . . . . . . . . . . . . . . . . . 5

1.4 Normal subgroups of division rings with involution $\ldots . . . . . . .10$

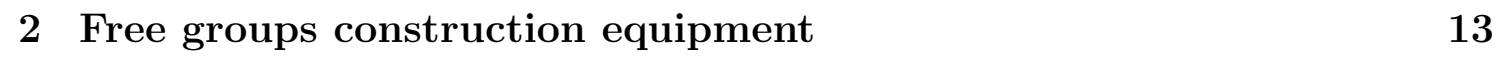

2.1 Valuations and combinatorial arguments . . . . . . . . . . . . . . 13

2.2 Valuations and algebraic arguments . . . . . . . . . . . . . . . 17

3 Free symmetric pairs in normal subgroups 23

3.1 Existence of non central symmetric elements . . . . . . . . . . . . 23

3.2 Main results . . . . . . . . . . . . . . . . . . . . 26

3.3 A note on characteristic 2 . . . . . . . . . . . . . . . . . . 44

\begin{tabular}{ll}
\hline Bibliography & 47
\end{tabular}

$\begin{array}{ll}\text { Index } & 51\end{array}$ 



\section{List of symbols}

\begin{tabular}{|c|c|}
\hline $\mathbb{Z}$ & ring of integer numbers \\
\hline $\mathbb{Q}$ & field of rational numbers \\
\hline$R^{\times}$ & collection of the invertible elements of the ring $R$ \\
\hline$R^{\mathrm{op}}$ & the opposite ring of the ring $R$ \\
\hline $\operatorname{id}_{R}$ & identity map of the ring $R$ \\
\hline$x^{u}$ & $u^{-1} x u$, where $x$ belongs to the ring $R$ and $u$ belongs to $R^{\times}$ \\
\hline$R[T]$ & $\begin{array}{l}\text { polynomial ring in the commutative indeterminate } T \text { with } \\
\text { coefficients in the ring } R\end{array}$ \\
\hline $\mathrm{Z}(R)$ & center of the ring (alternatively, the group) $R$ \\
\hline$\langle g, h\rangle$ & group generated by $g$ and $h$ \\
\hline$\langle G, H\rangle$ & group generated by the groups $G$ and $H$ \\
\hline $\mathrm{k}$ & arbitrary field \\
\hline $\mathbb{P}$ & prime subfield of $\mathrm{k}$ \\
\hline$D$ & arbitrary division ring \\
\hline$N$ & a normal subgroup of $D^{\times}$ \\
\hline$[G, G]$ & the commutator subgroup of the group $G$ \\
\hline$D(T)$ & quotient ring of $D[T]$ \\
\hline$D_{\alpha}$ & $D \otimes_{\mathbb{k}} \mathbb{k}_{k}(\alpha)$, where $\mathbb{k}=\mathrm{Z}(D)$ \\
\hline $\mathcal{S}_{\alpha}$ & set of polynomials whose evaluations in $\alpha$ are invertible \\
\hline$D_{\alpha}(T)$ & subring of $D(T)$ with denominators in $\mathcal{S}_{\alpha}$ \\
\hline$\pi_{\alpha}$ & evaluation map from $D_{\alpha}(T)$ onto $D_{\alpha}$ which sends $T$ to $\alpha$ \\
\hline $\operatorname{irr}(\alpha, \mathbb{k})$ & minimal polynomial of $\alpha$ over $\mathbb{k}$ \\
\hline$[K: \mathbb{k}]$ & degree of the field $K$ over $\mathbb{k}$ \\
\hline$f_{\mathrm{n}}(T)$ & "numerator" of a member $f(T)$ of $D(T)$ \\
\hline$f_{\mathrm{d}}(T)$ & "denominator" of a member $f(T)$ of $D(T)$ \\
\hline$*$ and $\bullet$ & involutions \\
\hline$S_{*}$ & collection of symmetric members of the set $S$ with respect \\
\hline & to an involution $*$ \\
\hline$\omega^{-*}$ & stands for $\left(\omega^{-1}\right)^{*}=\left(\omega^{*}\right)^{-1}$ \\
\hline
\end{tabular}




$\begin{array}{ll}\mathbb{H} & \text { quaternion algebra } \\ \mathbf{i} \text { and } \mathbf{j} & \text { quaternion generators, also } \mathbf{k}=\mathbf{i} \mathbf{j} \\ \mathrm{M}_{n}(R) & \text { ring of } n \times n \text { matrices with entries in the ring } R \\ \mathrm{GL}_{n}(R) & \text { multiplicative group of the invertible elements of } \mathrm{M}_{n}(R) \\ A(i, j) & \text { entry in the row } i \text { and column } j \text { of the matrix } A \\ A^{-\mathrm{T}} & \text { stands for }\left(A^{-1}\right)^{\top}=\left(A^{\top}\right)^{-1}, \text { where } A \text { is an invertible } \\ & \text { matrix } \\ \mathrm{I}_{m} & m \times m \text { identity matrix } \\ \operatorname{diag}\left(a_{1}, \ldots, a_{n}\right) & \text { matrix whose entries are } a_{1}, \ldots, a_{n} \text { along the main } \\ & \text { diagonal and zero elsewhere } \\ A \oplus B & \text { square matrix whose blocks along the main diagonal are } \\ & A \text { and } B \text { and whose off-diagonal blocks are zero } \\ v & \text { non archimedean valuation } \\ T_{v} & \left\{P \in \mathrm{GL}_{n}(\mathbb{k}) \mid v(P(i, j))<\infty \text { does not depend on } i, j\right\} \\ \mathrm{V}_{v}^{i} & \left\{\text { diag }\left(a_{1}, \ldots, a_{n}\right) \in \mathrm{GL}{ }_{n}(\mathbb{k}) \mid v\left(a_{i}\right)<v\left(a_{j}\right), j \neq i\right\} \\ \mathrm{V}_{v} & \bigcup_{i=1}^{n} \mathrm{~V}_{v}^{i}\end{array}$




\section{Introduction}

Exploring the existence of free objects within division rings is inserted in a broader context. It is tied up to the study of the algebraic structure of the multiplicative group of division rings and their subgroups. As it was outlined in [20], if $D$ is a noncommutative division ring with center $\mathbb{k}$, then, among other "is-notproperties", we have

(a) $D^{\times}$is not finite (Wedderburn [45]);

(b) $D^{\times}$is not torsion (Jacobson [28]);

(c) $\quad D^{\times}$is not solvable (Hua [27]); and

(d) $D^{\times} / \mathbb{k}^{\times}$is not torsion (Kaplansky [33]),

where $D^{\times}=D \backslash\{0\}$ and $\mathbb{k}^{\times}=\mathbb{k} \backslash\{0\}$ are the multiplicative groups of $D$ and $\mathbb{k}$, respectively. The main conjecture that gives perspective not only on our material, but also on what has been developed in the study of the existence of free objects in division rings is due to Lichtman. By the time he exhibited a counterexample to a more general statement of the Tits' Alternative [44], regarding matrix rings over a noncommutative division ring rather than over fields. The counterexample came in [36], when Lichtman proposed the following

Conjecture 1. The multiplicative group of a noncommutative division ring contains a non cyclic free group.

Even though a normal subgroup $N$ of $D^{\times}$(which is not contained in $\mathbb{k}$ ) is not necessarily closed under addition, it captures important properties of $D^{\times}$itself. In comparison to those properties of $D^{\times}$we presented in the previous paragraph, we have

(e) $\quad N$ has the same cardinality of $D^{\times}$, therefore it is not finite (Scott [43]);

(f) $\quad N$ is not torsion (Zalesskii [46]); 
(g) $\quad N$ is not solvable (Scott [43]); and

(h) $\quad N / \mathrm{Z}(N)$ is not torsion, provided that $\mathbb{k}$ is uncountable or $\operatorname{dim}_{\mathbb{k}} D<\infty$ (Herstein [25], Gonçalves [11]).

Taking into account whether $N$ contains a non cyclic free group, Lichtman presented the following

Theorem ([37], theorem 2). Let $N$ be a normal subgroup of the multiplicative group of a division ring. If $N$ contains a nonabelian nilpotent-by-finite subgroup, then it contains a non cyclic free group.

Regarding conjecture 1 in the context of studying the structure of subgroups of division rings, we were thus led to

Conjecture 2. A non central normal subgroup of the multiplicative group of a noncommutative division ring contains a non cyclic free group.

On one hand, such change of environment - passing from the multiplicative group of the whole division ring to an arbitrary non central normal subgroup - certainly hardens the task of proving the existence of free groups, since we are left solely with the multiplication. On the other hand, the existence of free groups in non central normal subgroups of division rings may be perceived to be bound up with the fact that multiplication is not commutative in such scenario.

A great deal of work has been done on the matter, offering affirmative answers, with respect to conjectures 1 and 2 , for several families of division rings. We highlight a remarkable result attained by Gonçalves in the following

Theorem ([11], theorem 2.1). A non central subnormal subgroup of the multiplicative group of a finite dimensional division ring contains a non cyclic free group.

It is also worthy to register a result due to Chiba, which is an improvement of ([40], theorem 1), as we see in the following

Theorem ([4], theorem 2). Let $D$ be a noncommutative division ring with uncountable center $\mathbb{k}$. Then $D^{\times}$contains a free non cyclic subgroup whose cardinality is equal to that of $\mathrm{k}$.

We assume from now on that $D$ has characteristic unequal to 2 and is endowed with an involution $*$. Although the collection $D_{*}$ of its symmetric members is not a group, it has important properties, such as

(i) $\quad D_{*}^{\times}$is not torsion (Herstein and Susan Montgomery [26]); 
(j) If $D$ is not the quaternion algebra endowed with the symplectic involution, then $D_{*}^{\times} / \mathbb{k}^{\times}$is not torsion (Chacron [3]); and

(k) If $D_{*}$ is not contained in $\mathrm{k}$, then no proper division subring of $D$ contains $D_{*}($ Dieudoneé [7]).

In [22], the authors established a new line of inquiry. Division rings, as many other families of algebras, are very often endowed with an involution. Therefore it seemed reasonable to frame the work on conjectures 1 and 2 regarding algebras with involution. In other words, the research on the existence of free groups within $D$ which are invariant under a given involution inevitably led to

Conjecture 3. Let $D$ be a division ring whose center has characteristic distinct from 2. Suppose that $D$ is endowed with an involution. Then $D^{\times}$contains symmetric and unitary free pairs.

Progress has been made upon the examination of conjecture 3 . Affirmative answers are found in [9, 18, from where we single out the results below.

Theorem ([18], theorem 1.1). Let $\mathbb{k}$ be a non absolute field of characteristic unequal to 2 and let $R$ be a noncommutative central simple $\mathrm{k}$-algebra with an involution * of the first kind. Then $R$ contains free symmetric pairs (except when $(R, *)$ is a quaternion algebra with the symplectic involution) and free unitary pairs (except when $(R, *)$ is a quaternion algebra with an orthogonal involution).

and

Theorem ([18], theorem 1.2). Let $\mathrm{k}$ be a non absolute field of characteristic unequal to 2 and let $R$ be a noncommutative central simple $\mathrm{k}$-algebra with an involution $*$ of the second kind. Then $R$ contains free symmetric and unitary pairs.

In combination, these theorems above extend ([9], lemma 2.6).

Recently, the research has been pushed forward in [20] - which is also a panorama on the subject - and in [8]. In [10], the concern with free symmetric pairs is given new contours, as the authors provided circumstances under which they exist in non central normal subgroups of division rings. Among other results, they presented

Theorem ([10], theorem 1.4). Let $D$ be a division ring with center $\mathbb{k}$ of characteristic different from 2 , with a $\mathrm{k}$-involution $*$. Let $N$ be a normal subgroup of $D^{\times}$ and assume that $N$ contains a torsion-free nilpotent-by-finite subgroup $G$ which is not abelian-by-finite. If both $G$ and $N$ are invariant under $*$, then $N$ contains a free symmetric pair with respect to $*$. 
Furthermore, regarding non central normal subgroups of division rings with involution, it is worthy to cite the remarkable and recently attained theorem below, which stands as an involutorial version of Lichtman's earlier mentioned result ([37], theorem 2).

Theorem ([16], theorem 1.2). Let $D$ be a division ring with central subfield $\mathrm{k}$ of characteristic distinct from 2 and let $*$ be a $\mathrm{k}$-involution in D. Now suppose $N$ is a normal subgroup of $D^{\times}$that contains a nonabelian *-stable subgroup $G$ that is either torsion free polycyclic-by-finite but not abelian-by-finite, or is finite of odd order. Then $N$ contains a free symmetric pair.

Let $D$ be a noncommutative division ring of characteristic unequal to 2 , endowed with an involution $*$. Let $N$ be a normal subgroup of $D$ not contained in $\mathbb{k}$. We shall prove the following

Proposition 1.4.1. $N$ contains a non central normal subgroup of $D^{\times}$which is invariant under $*$,

which spares us the assumption that non central normal subgroups are invariant under the given involution. Regarding conjectures 1, 2 and 3, taking into account the properties of $D^{\times}$captured by $N$, the properties of the collection of symmetric members of $D$ and proposition 1.4.1, it is thus reasonable to put our work in perspective regarding the following

Conjecture 4. Let $D$ be a division ring with center $\mathbb{k}$, a field whose characteristic is distinct from 2. Suppose that $D$ is endowed with an involution. A non central normal subgroup $N$ of $D^{\times}$contains symmetric and unitary free pairs.

With the present material, our objective is to give support to conjecture 4, mostly focused on free symmetric pairs.

We dedicate the first chapter to settle a minimal background for the whole text. We weave a web of some fundamental results in noncommutative ring theory and describe, as it has been done in a more general way by Amitsur in [1], a certain subring $D_{\alpha}(T)$ of the ring of fractions of the polynomial ring $D[T]$ and also an evaluation map which is defined in $D_{\alpha}(T)$. Besides the preliminary topics, we give attention to facts about involutions, especially those defined in quaternion algebras, and explain some important properties of normal subgroups of division rings (with involution), which are derived from Scott's theorems ([43], theorems 2 and 3), with special attention to the proposition 1.4.1 aforementioned. We omit (hopefully, the minimum possible) what we expect the reader to be familiar with, since it is an 
exposition of rather classical results and definitions, specially in what concerns facts and terminology of group theory. The details may be found in [5, 23, 35, 39, 42.

The second chapter is somehow a bridge between the first and the third ones. There we provide rather technical results that will aid us to study the existence of free symmetric pairs in non central normal subgroups of division rings with involution. The foremost subject we explore in chapter 2 is the concept of non archimedean valuation $v: \mathbb{k} \rightarrow G$ of a field $\mathbb{k}$ into an ordered abelian group $G$ and how it applies to the construction of free groups. We give a careful proof of a version of the main theorem in 38] which fits in with our goals, which affords us valuations into $G=\mathbb{Z}$. In addition, regarding the collections

$$
\begin{aligned}
\mathrm{T}_{v} & =\left\{P \in \mathrm{GL}_{n}(\mathrm{k}) \mid v(P(i, j)) \text { is finite and independent of } i \text { and } j\right\} \\
\mathrm{V}_{v}^{i} & =\left\{\operatorname{diag}\left(a_{1}, \ldots, a_{n}\right) \in \mathrm{GL}_{n}(\mathbb{k}) \mid v\left(a_{i}\right)<v\left(a_{j}\right), \text { for all } j \text { in }\{1, \ldots, n\} \backslash\{i\}\right\} \\
\mathrm{V}_{v} & =\bigcup_{i=1}^{n} \mathrm{~V}_{v}^{i},
\end{aligned}
$$

we insert in what has been serving as a "tool box" for the construction of free groups the following

Lemma 2.1.3. Let $v$ be a non archimedean valuation of a field $\mathrm{k}$ and $n$ be an integer number greater than 1 . Let $A, B, P$ and $Q$ be a members of $\mathrm{GL}_{n}(\mathrm{k})$ such that

(i) $A, B$ and their inverses belong to $\mathrm{V}_{v}$;

(ii) $P, Q$ and their inverses belong to $\mathrm{T}_{v}$; and

(ii) either $Q P=P Q=I$ or both $Q P$ and its inverse belong to $\mathrm{T}_{v}$,

then $\{A P B Q, P B Q A\}$ is a free pair.

We recommend [34, 6] for detailed explanation of field theory, [32] for algebraic number theory and [41] for valuation theory.

Chapter 3 is the most important one. It is reserved to our main theorems. We offer, among other results, the following

Theorem 3.2.2, Let $D$ be a noncommutative division ring of finite dimension over its center $\mathbb{k}$, an uncountable field whose characteristic is distinct from 2 . Let $*$ be a $\mathbb{k}$-involution in $D$ and $N$ be a non central normal subgroup of $D^{\times}$. Unless $D$ is a quaternion algebra endowed with the symplectic involution, $N$ contains a free symmetric pair. 
In order to prove theorem 3.2 .2 , we embed $D$ into $D_{\alpha}=D \otimes_{\mathbb{k}} \mathbb{k}(\alpha)$, where $\alpha$ is a separable algebraic element over $\mathbb{k}$ such that $D_{\alpha}$ is isomorphic to a full matrix ring $\mathrm{M}_{n}(\mathbb{k}(\alpha)) . D_{\alpha}$ is connected with $D(T)$ by means of the evaluation map which sends $T$ to $\alpha$. All such maps commute with $*$, which is naturally extended to $D(T)$ and to $D_{\alpha}$. Roughly speaking, we have enough room in $D_{\alpha}$ and the involution in $\mathrm{M}_{n}(\mathbb{k}(\alpha))$ is nicely described by means of conjugation and transposition. Free symmetric pairs are thus detected in $\mathrm{M}_{n}\left(\mathrm{k}(\alpha)\right.$ ) (equivalently, in $D_{\alpha}$ ) and then lifted to $D(T)$. A cancellation argument (lemma 2.2.2) finally applies to show that evaluation of $T$ in some member of $\mathbb{k}$ produces a free symmetric pair in $N$.

We give special attention to the fact that conjecture 4 is valid for division quaternion algebras with involutions of the first kind, which is presented in the following

Theorem 3.2.3. Let $\mathbb{k}$ be a field of characteristic distinct from 2 and $\mathbb{H}$ be a division quaternion $\mathrm{k}$-algebra. Let $N$ be a non central normal subgroup of $\mathbb{H}^{\times}$. Regarding an involution $*$ of the first kind in $\mathbb{H}$, the following assertions are true.

(i) If $*$ is orthogonal, then $N$ contains a free symmetric pair; and

(ii) if $*$ is symplectic, then $N$ contains a free unitary pair.

As it is widely known, on one hand, the unitary elements in $\mathbb{H}$ with respect to an orthogonal involution comprise an abelian subgroup of $\mathbb{H}^{\times}$. On the other hand, the symmetric elements of $\mathbb{H}$ always belong to $\mathbb{k}$. As to division quaternion algebras with involutions of the second kind, we offer

Theorem 3.2.5. Let $\mathbb{H}$ be a division quaternion algebra with center $\mathbb{k}$, a field whose characteristic is distinct from 2. Let $N$ be a non central normal subgroup of $\mathbb{H}^{\times}$. If $\mathbb{H}$ is endowed with an involution * of the second kind, then $N$ contains a free symmetric pair.

We observe that, in combination, theorems 3.2.3 and 3.2.5 leave us closer to a generalization of ([9], lemma 2.6). The multiplicative relation among the quaternion generators spares us the embedding of $\mathbb{H}$ in a scalar extension, therefore we need no cancellation argument in order to prove theorems 3.2 .3 and 3.2 .5 and so no assumption is made on the cardinality of $\mathbb{k}$.

The following results may be considered as consequences of the theorems 3.2 .3 and 3.2.5. The strategy for their proofs is to detect a subring of $D$ which is a quaternion algebra with non central intersection with $N$. 
Theorem 3.2.7. Let $D$ be a noncommutative division ring of finite dimension over its center $\mathbb{k}$, a field whose characteristic $p$ is distinct from 2 . Let $*$ be a $\mathbb{k}$-involution in $D$ and $N$ be a non central normal subgroup of $D^{\times}$. If $N$ contains a non central symmetric element of finite multiplicative order, then $N$ contains a free symmetric pair.

Theorem 3.2.7 may be rebuilt without the assumption on the dimension of $D$ or on the kind of $*$ as in the following

Theorem 3.2.9. Let $D$ be a noncommutative division ring with center $\mathrm{k}$, a field whose characteristic $p$ is distinct from 2. Let $*$ be an involution in $D$ and $N$ be a non central normal subgroup of $D^{\times}$. We assume that $N$ contains a non central symmetric element $\omega$ of finite multiplicative order. If either (i) $p=0$; or (ii) $p>0$ and the degree of $\operatorname{irr}(\omega, \mathrm{k})$ is even; then $N$ contains a free symmetric pair.

Finally, regardless of the dimension of the division ring and the kind of the involution it is endowed with, we offer

Theorem 3.2.10, Let $D$ be a division ring with center $\mathrm{k}$, a field whose characteristic is distinct from 2. Let $*$ be an involution in $D$ and $N$ be a non central normal subgroup of $D^{\times}$. If $N$ contains a non central symmetric element $\omega$ such that $[\mathbb{k}(\omega): \mathbb{k}]=2$, then $N$ contains a free symmetric pair.

We end the third chapter with an example of a division ring whose center has characteristic 2 which is endowed with an involution of the first kind and whose symmetric elements have central squares. This explains why one should preferably deal with involutions in division rings with characteristic distinct from 2 in order to seek free symmetric pairs. 



\section{Chapter 1}

\section{Preliminaries}

\subsection{Minimal background}

Let $R$ be a simple ring. Its center $\mathbb{k}$ certainly is a field and its elements are called central. Furthermore, as it is usual, an invertible element of $R$ is said to be a unit. The collection of units of $R$, which is known to comprise a multiplicative group, is called the multiplicative group of $R$ and is denoted by $R^{\times}$.

In the study of division rings, we can imagine a "tectonic plate" of theorems over which major conclusions settle. Those fundamental results must of course include classic ones for non commutative rings, with special attention to the study of simple rings. We are to make extensive use of some of them, which we record below, beginning with the celebrated Skolem-Noether theorem.

Theorem 1.1.1 (e.g. [23], theorem 4.3.1). Let $R$ be a simple artinian ring with center $\mathbb{k}$ and let $A$ and $B$ be simple subrings of $R$ which contain $\mathbb{k}$ and are finitedimensional over it. If $\phi$ is an isomorphism from $A$ onto $B$ leaving $\mathbb{k}$ fixed elementwise, there then exists an invertible element $u$ in $R$ such that

$$
\phi(x)=x^{u}=u^{-1} x u,
$$

for every $x$ in $A$.

The Skolem-Noether theorem applies to the proofs of useful results for division rings. The first one we register is due to Wedderburn, widely known as his little theorem.

Theorem 1.1.2 (e.g. [23], application (3) of theorem 4.3.1). A finite division ring necessarily is commutative.

The second result which may be obtained with the aid of the Skolem-Noether is due to Dickson. 
Theorem 1.1.3 (e.g. [23], application (4) of theorem 4.3.1). Let $D$ be a division ring with center $\mathbb{k}$. If $x$ and $y$ are members of $D$ both of which are algebraic over $\mathbb{k}$, then they are conjugate in $D$ if and only if they have the same minimal polynomial over $\mathbb{k}$.

A normal subgroup of (the multiplicative group of) a division ring calls our attention whenever it contains non central elements. Such subgroups are plausibly called non central. In what concerns normal subgroups of a division ring, an argument that interlaces Wedderburn's little theorem and Cartan-Brauer-Hua theorem ([29], chapter VII, section 13) was presented by Scott in the remarkable

Theorem 1.1.4 ([43], theorem 2). Let $D$ be a division ring with center $\mathrm{k}$. Then $D^{\times} / \mathbb{k}^{\times}$contains no nontrivial abelian normal subgroup.

The way is thus clear for us to state a more objective result, which the following well known

Corollary 1.1.5. Let $D$ be a division ring. Any abelian normal subgroup of $D^{\times}$is central.

Proof: If $N$ is an abelian normal subgroup of $D^{\times}$, then $\left\langle N, \mathbb{k}^{\times}\right\rangle=N \mathbb{k}^{\times}$is also an abelian normal subgroup of $D^{\times}$, therefore $\left(N k^{\times}\right) / \mathbb{k}^{\times}$is an abelian normal subgroup of $D^{\times} / \mathbb{k}^{\times}$. By virtue of theorem 1.1.4. $\left(N \mathbb{k}^{\times}\right) / \mathbb{k}^{\times}$is trivial, which entails that $N$ is central.

Furthermore, with the aid of the Cartan-Brauer-Hua theorem, Scott proved the following

Theorem 1.1.6 ([4], theorem 3). The intersection of two non central normal subgroups of the multiplicative group of a division ring is non central.

The results we registered above have always been fruitful material. We are prepared to articulate them throughout our work.

\subsection{Extension of the evaluation map}

We consider a division ring $D$ with center $\mathrm{k}$ and a commutative indeterminate $T$ over $D$. It is immediately seen that the polynomial ring $D[T]$ is isomorphic to $D \otimes_{\mathbb{k}} \mathbb{k}[T]$ with respect to the rule that assigns $1 \otimes T^{k}$ to $T^{k}$, for each natural number $k$. Such map gives rise to a unique $D$-linear bijection from $D[T]$ onto $D \otimes_{\mathbb{k}} \mathbb{k}[T]$, which is readily seen to be multiplicative. 
Let $\alpha$ be an algebraic element over $\mathrm{k}$. We write $\varepsilon_{\alpha}$ to denote the homomorphism from $\mathbb{k}[T]$ onto $\mathbb{k}(\alpha)$ which maps $T$ to $\alpha$ and $D_{\alpha}$ to denote the ring $D \otimes_{\mathbb{k}} \mathbb{k}(\alpha)$. The composition of the isomorphism described in the previous paragraph with the map id $\otimes \varepsilon_{\alpha}: D \otimes_{\mathbb{k}} \mathbb{k}[T] \rightarrow D_{\alpha}$ determines a surjective homomorphism we shall denote by

$$
\pi_{\alpha}: D[T] \rightarrow D_{\alpha} \text { and } \pi_{\alpha}(f(T))=f(\alpha),
$$

for each member $f(T)$ of $D[T]$. We also refer to $\pi_{\alpha}$ as the evaluation map from $D[T]$ onto $D_{\alpha}$ which sends $T$ to $\alpha$.

We refer the reader to ([5], chapter 1 , section 3) and to ([39], chapter 4, section 4) for a detailed explanation of the Ore condition and for the construction and properties of the division ring $D(T)$ of fractions of the noetherian domain $D[T]$. Explicitly, whenever we are given an arbitrary member $f(T)$ of $D(T)$, there exist a "numerator" $f_{\mathrm{n}}(T)$ and a nonzero "denominator" $f_{\mathrm{d}}(T)$, both belonging to $D[T]$ and such that

$$
f(T)=f_{\mathrm{n}}(T) f_{\mathrm{d}}(T)^{-1}
$$

and it is clear that we are allowed to assume that $f_{\mathrm{n}}(T)$ and $f_{\mathrm{d}}(T)$ share no common right factor of positive degree in $D[T]$.

Our goal is to describe a certain extension $D_{\alpha}(T)$ of $D[T]$ within $D(T)$ and to extend $\pi_{\alpha}$ to $D_{\alpha}(T)$. Specifically, $D_{\alpha}(T)$ is determined within $D(T)$ so that an element $f(T)$ of $D[T]$ is invertible in $D_{\alpha}(T)$ whenever its image $f(\alpha)$ under $\pi_{\alpha}$ is invertible in $D_{\alpha}$. Therefore $D_{\alpha}(T)$ may be understood as the largest possible subring of $D(T)$ to which $\pi_{\alpha}$ admits an extension. It has been done in a more general manner in ([1], lemma 5), considering an arbitrary subfield of $\mathrm{k}$ rather than $\mathrm{k}$ itself and a matrix ring $\mathrm{M}_{n}(D[T])$ instead of $D[T]$. For the sake of completeness, we provide the explanation for the special case in the following

Lemma 1.2.1. Let $D$ be a division ring with center $\mathrm{k}$. Let $\alpha$ be an algebraic element over $\mathrm{k}$. Regarding the evaluation map $\pi_{\alpha}$ from $D[T]$ onto $D_{\alpha}$, the multiplicative set

$$
\mathcal{S}_{\alpha}=\{f(T) \in D[T] \mid f(\alpha) \text { is invertible }\}
$$

verifies the Ore condition, therefore

$$
D_{\alpha}(T)=\left\{f(T) g(T)^{-1} \mid f(T) \in D[T], g(T) \in \mathcal{S}_{\alpha}\right\}
$$

is a subring of $D(T)$, to which $\pi_{\alpha}$ is extended, obeying the well-defined rule

$$
\pi_{\alpha}\left(f(T) g(T)^{-1}\right)=f(\alpha) g(\alpha)^{-1}
$$

and $f(\alpha) g(\alpha)^{-1}$ is invertible if and only if $f(T) g(T)^{-1}$ is invertible in $D_{\alpha}(T)$, for every $f(T)$ in $D[T]$ and $g(T)$ in $S_{\alpha}$. 
Proof: We fix arbitrary polynomials $f(T)$ in $\mathcal{S}_{\alpha}$ and $g(T)$ in $D[T]$ and proceed to show that the sets $f(T) D[T]$ and $g(T) \mathcal{S}_{\alpha}$ have nonempty intersection, which precisely is the (right) Ore condition for $\mathcal{S}_{\alpha}$. Indeed, $f(T)$ certainly is nonzero and so we write $h(T)$ to denote the member

$$
f(T)^{-1} g(T)
$$

of $D(T)$, which yields, regarding the "numerator" $h_{\mathrm{n}}(T)$ and the "denominator" $h_{\mathrm{d}}(T)$ of $h(T)$,

$$
f(T) h_{\mathrm{n}}(T)=g(T) h_{\mathrm{d}}(T)
$$

Since $h_{\mathrm{n}}(T)$ and $h_{\mathrm{d}}(T)$ share no common right factor of positive degree in $D[T]$, there exist $p(T)$ and $q(T)$ in $D[T]$ such that

$$
p(T) h_{\mathrm{n}}(T)+q(T) h_{\mathrm{d}}(T)=1 \text {. }
$$

In light of the equation (1.1), the arguments in this paragraph will be complete after we have proved that $h_{\mathrm{d}}(T)$ belongs to $\mathcal{S}_{\alpha}$. In agreement with ([5], lemma 6.4.5), $D_{\alpha}$ is isomorphic to a matrix ring over a division ring, therefore each one of its elements either is invertible or is a two-sided zero divisor. It means that our task may be reduced to show that $h_{\mathrm{d}}(\alpha)$ is not a left zero divisor, which we claim to be true. Let $k(T)$ be an element of $D[T]$ such that

$$
h_{\mathrm{d}}(\alpha) k(\alpha)=0
$$

By virtue of the equations (1.1), since $f(\alpha)$ is invertible, we have

$$
h_{\mathrm{n}}(\alpha) k(\alpha)=f(\alpha)^{-1} g(\alpha) h_{\mathrm{d}}(\alpha) k(\alpha)=0
$$

therefore, by virtue of the equation 1.2 , it follows

$$
k(\alpha)=p(\alpha) h_{\mathrm{n}}(\alpha) k(\alpha)+q(\alpha) h_{\mathrm{d}}(\alpha) k(\alpha)=0 .
$$

The claim is thus proved.

It is thus clear that the remaining of the statement derives from the universal property of localization.

Plausibly, if we refer to $f(T)$ as a member of $D_{\alpha}(T)$, it is understood that its "denominator" $f_{\mathrm{d}}(T)$ belongs to $\mathcal{S}_{\alpha}$. We emphasize that $D$ is a subring of $D_{\alpha}(T)$ which is identically mapped into $D_{\alpha}$ by $\pi_{\alpha}$. 


\subsection{Involutions}

Let $R$ be a simple ring with center $\mathbb{k}$. An involution in $R$ is an additive, anti-multiplicative and self-inverse map $*: R \rightarrow R$. With respect to $*$, an element $x$ of $R$ is said to be symmetric if $x^{*}=x$; skew-symmetric, if $x^{*}=-x$; and unitary, if $x$ is invertible and $x^{*}=x^{-1}$. Of course, no distinction is to be made between symmetry and skew-symmetry properties whenever $\mathrm{k}$ has characteristic 2 . For an arbitrary subset $S$ of $R$, we denote

$$
S_{*}=\left\{x \in S \mid x^{*}=x\right\} .
$$

It is promptly seen that $*$ acts as an automorphism of $\mathbb{k}$ and, since $*$ is its own inverse, either $\mathbb{k}_{*}=\mathbb{k}$ and $*$ is said to be a $\mathrm{k}$-involution (alternatively, an involution of the first kind) or $\mathbb{k}_{*}$ is a proper subfield of $k_{\text {such }}$ that $\left[\mathbb{k}: \mathbb{k}_{*}\right]=2$ and $*$ is said to be an involution of the second kind.

Let $T$ be a commutative indeterminate over a division ring $D$ with center $\mathbb{k}$. Regarding an involution $*$ in $D$, we shall prove that $*$ extends to an involution of the same kind in $D(T)$. Indeed, the rule that maps each $f(T)=\sum \omega_{i} T^{i}$ in $D[T]$ to

$$
f(T)^{*}=\sum \omega_{i}^{*} T^{i}
$$

defines an injective homomorphism from $D[T]$ into $D(T)^{\text {op }}$ and it is clear that the image of each nonzero polynomial is invertible. In light of ([5], proposition 1.3.4), it follows that $*$ extends to an isomorphism from $D(T)$ onto $D(T)^{\text {op }}$. Equivalently, * extends to an involution of $D(T)$, which therefore obeys the rule that maps $f(T)$ to

$$
\left(f_{\mathrm{d}}(T)^{*}\right)^{-1} f_{\mathrm{n}}(T)^{*}
$$

for each $f(T)$ in $D(T)$, regardless of how $f_{\mathrm{n}}(T)$ or $f_{\mathrm{d}}(T)$ have been chosen. In order to certify that the kind of the extended involution (which we also denote by $*$ ) is the same of the original one, we observe that $\mathrm{Z}(D(T))=\mathbb{k}(T)$ (e.g. [5], proposition 2.1.5) and, since it is manifest that $\mathbb{k}_{(}(T)_{*}=\mathbb{k}_{*}(T)$, we have

$$
\left[\mathbb{k}(T): \mathbb{k}(T)_{*}\right]=\left[\mathbb{k}(T): \mathbb{k}_{*}(T)\right]=\left[\mathbb{k}: \mathbb{k}_{*}\right]
$$

We assume that $*$ is of the first kind and fix an element $\alpha$ which is algebraic element over $\mathrm{k} . D$ is canonically embedded in $D_{\alpha}$ and the map $* \otimes$ id is promptly seen to be a $\mathbb{k}_{k}(\alpha)$-involution of $D_{\alpha}$ which also extends $*$ and so we briefly denote it by $*$. If we are given an arbitrary member $f(T)^{*}=\sum \omega_{i} T^{i}$ in $\mathcal{S}_{\alpha}$, we have

$$
\pi_{\alpha}\left(f(T)^{*}\right)=\sum \omega_{i}^{*} \otimes \alpha^{i}=\left(\sum \omega_{i} \otimes \alpha^{i}\right)^{*}=f(\alpha)^{*},
$$


which clearly belongs to $\mathcal{S}_{\alpha}$. It thus follows that $\mathcal{S}_{\alpha}$ is invariant under $*$, therefore $D_{\alpha}(T)$ is invariant under $*$ and $\pi_{\alpha}$ obeys the identity

$$
\pi_{\alpha}\left(f(T)^{*}\right)=f(\alpha)^{*}
$$

for every $f(T)$ in $D_{\alpha}(T)$.

We turn our attention to the simplest possible non commutative division algebra. Let $\mathbb{k}$ be a field of characteristic distinct from 2 . A $\mathbb{k}$-algebra $\mathbb{H}$ is said to be a quaternion algebra if it is generated over $\mathbb{k}$ by members $\mathbf{i}$ and $\mathbf{j}$ whose squares belong to $\mathbb{k}^{\times}$and such that $\mathbf{j} \mathbf{i}=-\mathbf{i} \mathbf{j}$. It is widely known that it is also possible to define quaternion algebras over fields of characteristic 2, to which we shall briefly address in a further moment. In those circumstances, we say that $\mathbf{i}$ and $\mathbf{j}$ are quaternion generators. $\mathbb{H}$ is said to be a division quaternion algebra if it is also a division ring. Routinely computation reveals that $\{1, \mathbf{i}, \mathbf{j}, \mathbf{i} \mathbf{j}\}$ is a basis of $\mathbb{H}$ over $\mathbb{k}$, therefore such field precisely is its center even if $\mathbb{H}$ fails to be a division ring. As usual, we denote $\mathbf{k}=\mathbf{i} \mathbf{j}$. An involution $*$ of $\mathbb{H}$ is said to be of type $\mathbf{I}$ if every pair of quaternion generators is comprised by skew-symmetric elements. Otherwise, * is said to be of type II.

It is rather important to make distinction between involutions of type I and II in quaternion algebras. Indeed, an arbitrary division quaternion algebras endowed with an involution of type II admits a somehow nice pair of generators, as it is verified in the following

Lemma 1.3.1 (9], lemma 2.5). A division quaternion algebra endowed with an involution * of type II contains a pair of symmetric quaternion generators.

Proof: We regard a quaternion $\mathbb{k}$-algebra $\mathbb{H}$ endowed with an involution $*$ and assume that all its symmetric members are central. If $\mathbf{i}$ and $\mathbf{j}$ are quaternion generators of $\mathbb{H}$, then $\mathbf{i}^{2}, \mathbf{j}^{2}, \mathbf{i}+\mathbf{i}^{*}, \mathbf{j}+\mathbf{j}^{*}, \mathbf{i}^{*} \mathbf{i}$ and $\mathbf{j}^{*} \mathbf{j}$ belong to $\mathbb{k}$ and so

$$
0=\mathbf{i}^{2}-\left(\mathbf{i}+\mathbf{i}^{*}\right) \mathbf{i}+\mathbf{i}^{*} \mathbf{i} \text { and } 0=\mathbf{j}^{2}-\left(\mathbf{j}+\mathbf{j}^{*}\right) \mathbf{j}+\mathbf{j}^{*} \mathbf{j}
$$

are dependence relations of the sets $\{1, \mathbf{i}\}$ and $\{1, \mathbf{j}\}$ over $\mathbb{k}$. Necessarily $\mathbf{i}^{*}=-\mathbf{i}$ and $\mathbf{j}^{*}=-\mathbf{j}$. If we assume that $*$ is an involution of type II, it entails that $\mathbb{H}_{*}$ is not contained in $\mathrm{k}$.

We fix quaternion generators $\mathbf{i}$ and $\mathbf{j}$ of $\mathbb{H}$, whose squares we respectively denote by $a$ and $b$. Let $\omega_{0}=\alpha+\beta \mathbf{i}+\gamma \mathbf{j}+\delta \mathbf{k}$ be a non central symmetric element of $\mathbb{H}$. We have

$$
\omega_{0}^{2}-2 \alpha \omega_{0}+\left(\alpha^{2}-a \beta^{2}-b \gamma^{2}+a b \delta^{2}\right)=0
$$


hence

$$
\omega_{0}^{2}-2 \alpha^{*} \omega_{0}+\left(\alpha^{2}-a \beta^{2}-b \gamma^{2}+a b \delta^{2}\right)^{*}=0,
$$

whence

$$
\omega_{0}^{2}-\left(\alpha+\alpha^{*}\right) \omega_{0}+\frac{\left(\alpha^{2}-a \beta^{2}-b \gamma^{2}+a b \delta^{2}\right)+\left(\alpha^{2}-a \beta^{2}-b \gamma^{2}+a b \delta^{2}\right)^{*}}{2}=0 .
$$

It thus follows that

$$
\omega=\omega_{0}-\frac{\alpha+\alpha^{*}}{2}
$$

is a non central symmetric member of $\mathbb{H}$ whose square accordingly is symmetric and belongs to $\mathbb{k}^{\times}$, therefore $\omega$ and $-\omega$ shares the same minimal polynomial over $\mathbb{k}$ and, by virtue of theorem 1.1 .3 , there exists $\theta$ in $\mathbb{H}^{\times}$such that $\theta \omega=-\omega \theta$. Consequently, $\{1, \omega, \theta, \omega \theta\}$ is a basis of $\mathbb{H}$ over $\mathbb{k}$ and $\theta^{2}$ belongs to $\mathbb{k}^{\times}$. If $\theta^{*}=\theta$, then $\omega$ and $\theta$ are symmetric quaternion generators. Otherwise, $\omega$ and $\omega^{-1}\left(\theta-\theta^{*}\right)$ are symmetric quaternion generators.

Moreover, we have the following result concerning involutions of type $\mathrm{I}$ in quaternion division algebras, which also appears in [9].

Proposition 1.3.2. An involution * of type I of a division quaternion $\mathbb{k}$-algebra $\mathbb{H}$ necessarily is of the first kind.

Proof: We fix a pair $\left\{\mathbf{i}_{0}, \mathbf{j}_{0}\right\}$ of quaternion generators of $\mathbb{H}$ and an arbitrary member $\lambda_{0}$ in $\mathrm{k}$ and denote

$$
\lambda=\lambda_{0}-\lambda_{0}^{*}, \quad \mathbf{i}=\lambda \mathbf{i}_{0} \text { and } \mathbf{j}=\lambda \mathbf{j}_{0}
$$

therefore $\mathbf{i}^{2}=\lambda^{2} \mathbf{i}_{0}^{2}$ and $\mathbf{j}^{2}=\lambda^{2} \mathbf{j}_{0}^{2}$ belong to $\mathbb{k}, \mathbf{j} \mathbf{i}=-\mathbf{i} \mathbf{j}, \mathbf{i}^{*}=\mathbf{i}$ and $\mathbf{j}^{*}=\mathbf{j}$. Since $*$ is an involution of type $\mathrm{I}$, we must conclude that $\mathbf{i}$ and $\mathbf{j}$ are not quaternion generators of $\mathbb{H}$, which entails $\lambda=0$ and so $\lambda_{0}^{*}=\lambda_{0}$. The result thus follows.

An involution of the first kind of a division quaternion algebra is said to be symplectic, if it is of type I, and orthogonal, if it is of type II. It is straightforward to verify that symmetric elements with respect to a symplectic involution are central, whereas unitary elements with respect to an orthogonal involution comprise an abelian multiplicative group. In agreement with proposition 1.3 .2 , involutions of the second kind in division quaternion algebras are always of type II.

By a non absolute field, one understands a field which is not an algebraic extension of a finite field. For further references, we claim that if $\mathbb{H}$ is a division quaternion algebra with center $\mathbb{k}$, then $\mathbb{k}$ necessarily is non absolute. Indeed, the characteristic of $\mathbb{k}$ either is zero (therefore $\mathbb{k}$ is non absolute) or is a prime number $p$ greater than 3 . In the latter case, the prime subfield $\mathbb{P}$ of $\mathbb{k}$ is finite (it has exactly 
$p$ elements). We fix quaternion generators $\mathbf{i}$ and $\mathbf{j}$ of $\mathbb{H}$ and denote their squares respectively by $a$ and $b$. Therefore, the $\mathbb{P}(a, b)$-algebra $\mathbb{H}_{0}$ generated by $\mathbf{i}$ and $\mathbf{j}$ is a division quaternion algebra. In light of Wedderburn's little theorem, $\mathbb{H}_{0}$ is infinite and, since it is finite dimensional over $\mathbb{P}(a, b)$, this field is also infinite, which means that at least one among $a$ and $b$ is transcendental over $\mathbb{P}$. With greater reason, $\mathbb{k}$ contains an element which is transcendental over any of its finite subfields, as claimed.

We close this section describing how an arbitrary involution of the first kind of a matrix ring over a field whose characteristic is distinct from 2 may be easily understood, which is in fact a very particular case of a well known theorem of Kaplansky (e.g. [2], theorem 4.6.8) for involutions in primitive rings with minimal ideals.

Proposition 1.3.3. Let $L$ be a field whose characteristic is distinct from 2. For a given integer number $n$ greater than 1 , we assume that $\mathrm{M}_{n}(L)$ is endowed with an $L$-involution *. There then exist $U$ and $V$ in $\mathrm{GL}_{n}(L)$ such that the rule that maps A to

$$
A^{\bullet}=U^{-1} A^{\top} U
$$

is an L-involution in $\mathrm{M}_{n}(L)$ and

$$
A^{V \bullet}=A^{* V}
$$

for every $A$ in $\mathrm{M}_{n}(L)$. Furthermore, either $U$ is diagonal or there exists a positive integer number $m$ such that $n=2 m$ and $U$ may be written in the block form

$$
U=\left[\begin{array}{cc}
0 & -\mathrm{I}_{m} \\
\mathrm{I}_{m} & 0
\end{array}\right] .
$$

Explicitly, if $A$ is a member of $\mathrm{M}_{n}(L)$ written in block form

$$
A=\left[\begin{array}{ll}
B & C \\
E & F
\end{array}\right],
$$

with $B, C, E$ and $F$ in $\mathrm{M}_{m}(L)$, then

$$
A^{\bullet}=\left[\begin{array}{cc}
F^{\top} & -C^{\top} \\
-E^{\top} & B^{\top}
\end{array}\right] .
$$

Proof: Since the transpose is itself an $L$-involution in $\mathrm{M}_{n}(L)$, the rule that maps each $A$ in $\mathrm{M}_{n}(L)$ to $\left(A^{*}\right)^{\top}$ is an $L$-automorphism. In agreement with the SkolemNoether theorem, there exists a matrix $H$ in $\mathrm{GL}_{n}(L)$ such that

$$
\left(A^{*}\right)^{\top}=H A H^{-1}
$$


hence

$$
A^{*}=H^{-\top} A^{\top} H^{\top},
$$

for every $A$ in $\mathrm{M}_{n}(L)$. We have

$$
A=A^{* *}=H^{-\mathrm{\top}}\left(H^{-\top} A^{\top} H^{\top}\right)^{\top} H^{\top}=\left(H^{-\top} H\right) A\left(H^{-\top} H\right)^{-1}
$$

for every matrix $A$ in $\mathrm{M}_{n}(L)$, hence $H^{-\mathrm{T}} H$ lies in the center of $\mathrm{M}_{n}(L)$, whence there must exist $\beta$ in $L$ such that $H^{\top}=\beta H$. It follows that

$$
H=H^{\top \mathrm{\top}}=(\beta H)^{\top}=\beta H^{\top}=\beta^{2} H .
$$

Since $H$ is invertible and the characteristic of $L$ is unequal to 2 , either $\beta=1$ or $\beta=-1$. In both cases, equation

$$
A^{*}=H^{-1} A^{\top} H
$$

holds. We separately analyze them ahead.

Case $\beta=1$. $H$ is a non singular matrix such that $H^{\top}=H$. As it is well known (e.g. [30], chapter $\mathrm{V}$, section 9), there exists $V$ in $\mathrm{GL}_{n}(L)$ such that $U=V^{\top} H V$ is a diagonal matrix. Since $U$ is non singular and $U^{\top}=U$, the rule that assigns, to each $A$ in $\mathrm{M}_{n}(L)$,

$$
A^{\bullet}=U^{-1} A^{\top} U
$$

is an $L$-involution in $\mathrm{M}_{n}(L)$. Therefore

$$
A^{* V}=V^{-1} A^{*} V=V^{-1}\left(H^{-1} A^{\top} H\right) V=U^{-1}\left(V^{-1} A V\right)^{\top} U=\left(V^{-1} A V\right)^{\bullet}=A^{V \bullet},
$$

for all $A$ in $\mathrm{M}_{n}(L)$.

Case $\beta=-1$. Now $H$ is a non singular matrix such that $H^{\top}=-H$. As it is well known (e.g. [30], chapter $\mathrm{V}$, section 10) there exist a positive integer number $m$ such that $n=2 m$ and a matrix $V$ in $\operatorname{GL}_{n}(L)$ such that $U=V^{\top} H V$ is written in block form

$$
U=\left[\begin{array}{cc}
0 & -\mathrm{I}_{m} \\
\mathrm{I}_{m} & 0
\end{array}\right] .
$$

Since $U$ is invertible and $U^{\top}=-U$, we may consider the $L$-involution that maps each $A$ in $\mathrm{M}_{n}(L)$ to

$$
A^{\bullet}=U^{-1} A^{\top} U
$$

As it has been computed in the previous case, $A^{* V}=A^{V \bullet}$, for all $A$ in $\mathrm{M}_{n}(L)$. 


\subsection{Normal subgroups of division rings with in- volution}

Let $D$ be a division ring which is algebraic over its center $\mathbb{k}$. If $D$ is endowed with an involution $*$ of the first kind, it is immediately verified that, for each $\omega$ in $D$,

$$
\operatorname{irr}(\omega, \mathbb{k})=\operatorname{irr}\left(\omega^{*}, \mathbb{k}\right) .
$$

In light of theorem 1.1.3, $\omega$ and $\omega^{*}$ are conjugate in $D$. It thus follows that a normal subgroup $N$ of $D^{\times}$is invariant under $*$. If $*$ is not assumed to fix $\mathbb{k}$ elementwise or $D$ is not algebraic over $\mathbb{k}$, we are not allowed to use the referred theorem of Dickson in order to conclude that $N$ is invariant under $*$. Nevertheless, for an arbitrary division ring endowed with an involution, we have the following

Proposition 1.4.1. Let $N$ be a non central normal subgroup of the multiplicative group of a division ring $D$ endowed with an involution $*$. Then $N$ contains a non central normal subgroup $N_{0}$ of $D^{\times}$which is invariant under $*$.

Proof: It is readily seen that $N^{*}=\left\{\omega^{*} \mid \omega \in N\right\}$ is a non central normal subgroup of $D^{\times}$. By virtue of theorem 1.1.6, $N_{0}=N \cap N^{*}$ is a non central normal subgroup of $D^{\times}$which manifestly is invariant under $*$.

The following proposition, which is a consequence of a well known theorem of Brauer for central simple algebras, is a key tool in the study of involutions of the first kind in finite dimensional division rings.

Proposition 1.4.2. Let $D$ be a division ring of finite dimension over its center $\mathbb{k}$, a field whose characteristic is distinct from 2 . If $D$ is endowed with a $\mathrm{k}$-involution, then the degree of the minimal polynomial of an arbitrary element of $D$ is a power of 2 . In particular, every member of $D$ is separable over $\mathbb{k}$.

Proof: A $k$-involution in $D$ precisely is a $\mathrm{k}$-isomorphism from $D$ onto its opposite ring $D^{\text {op }}$, therefore the class of $D$ in the Brauer group of $\mathbb{k}$ has order 2 . In light of ([23], lemma 4.4.5), there exists a non negative integer $n$ such that $\operatorname{dim}_{\mathbb{k}} D=2^{n}$. With greater reason, the degree of the minimal polynomial of an arbitrary member of $D$ over $\mathbb{k}$ divides $2^{n}$ and so is a power of 2 . Since $\mathbb{k}$ has characteristic distinct from 2, we conclude that every element of $D$ is separable over $\mathbb{k}$.

In a further moment, we shall prove that a non central normal subgroup of a finite dimensional division ring endowed with an involution of the first kind and whose center has characteristic unequal to 2 contains a non central symmetric element. The proof must begin with a fixed element in the normal subgroup whose 
square is non central. The following straightforward result provides what we need in its part (ii) and its part (i) shows that there is no ambiguity in referring to a non central element of a normal subgroup of a division ring - if it lies outside the center of the division ring, it also lies outside the center of the normal subgroup.

Proposition 1.4.3. Let $D$ be a noncommutative division ring with center $\mathbb{k}$. For an arbitrary normal subgroup $N$ of $D^{\times}$, the following assertions are true.

(i) The center of $N$ is $N \cap \mathbb{k}$; and

(ii) if $N$ is non central, then it contains an element whose square lies outside $\mathbb{k}$.

Proof: As a characteristic subgroup of $N, \mathrm{Z}(N)$ is an abelian normal subgroup of $D^{\times}$and, in accordance with the corollary 1.1 .5 , must be contained in $\mathbb{k}$. This proves (i). In order to prove (ii), we assume that the square of every element in $N$ belongs to k. Consequently, such property is also valid for the normal subgroup $\left\langle N, \mathbb{k}^{\times}\right\rangle=N \mathbb{k}^{\times}$of $D^{\times}$, whose center is, in light of the assertion (i), $\mathrm{Z}\left(N \mathbb{k}^{\times}\right)=\mathbb{k}^{\times}$. For arbitrary members $\omega$ and $\theta$ of $N \mathbb{k}^{\times}$, there exist $\lambda$ and $\mu$ in $\mathbb{k}$ such that $\omega^{-1}=\lambda \omega$ and $\theta^{-1}=\mu \theta$, hence

$$
\omega^{-1} \theta^{-1} \omega \theta=(\lambda \omega)(\mu \theta) \omega \theta=\lambda \mu(\omega \theta)^{2}
$$

and we conclude that the commutator subgroup of $N \mathrm{k}^{\times}$is contained in its center. Consequently, $\left(N k^{\times}\right) / k^{\times}$is an abelian normal subgroup of $D^{\times} / \mathbb{k}^{\times}$. By virtue of theorem 1.1.4, it follows that $N$ is central, which finishes this proof. 



\section{Chapter 2}

\section{Free groups construction equipment}

\subsection{Valuations and combinatorial arguments}

Let $v$ be a non archimedean valuation of a field $\mathrm{k}$ into an abelian ordered group. By definition, inequality

$$
\min \{v(\alpha), v(\beta)\} \leqslant v(\alpha+\beta)
$$

holds for every $\alpha$ and $\beta$ in $\mathrm{k}$. A simple but useful result directly derived from the defining properties of a non archimedean valuation is that such inequality may be replaced by an equality whenever $v(\alpha)$ and $v(\beta)$ are distinct. For a fixed natural number $n$ greater than 1 , we thus have enough motivation to single the following subsets out of $\mathrm{GL}_{n}(\mathrm{k})$ :

$$
\begin{aligned}
\mathrm{T}_{v} & =\left\{P \in \mathrm{GL}_{n}(\mathbb{k}) \mid v(P(i, j)) \text { is finite and independent of } i \text { and } j\right\} \\
\mathrm{V}_{v}^{i} & =\left\{\operatorname{diag}\left(a_{1}, \ldots, a_{n}\right) \in \mathrm{GL}_{n}(\mathbb{k}) \mid v\left(a_{i}\right)<v\left(a_{j}\right), \text { for all } j \text { in }\{1, \ldots, n\} \backslash\{i\}\right\} \\
\mathrm{V}_{v} & =\bigcup_{i=1}^{n} \mathrm{~V}_{v}^{i} .
\end{aligned}
$$

Regarding the sets above, a rather general result ([22], lemma 6) describes some relationships among analogous collections in the context of crossed products. Since we are to deal with finite dimensional division rings, right regular representation - as it is defined and exemplified in [8] - shall establish the connection we need between the members of the division ring and matrices with entries in a field. It is convenient, therefore, to consider the following 
Lemma 2.1.1 ([17], lemma 2.3). Let $v$ be a non archimedean valuation of a field $\mathrm{k}$ and let $n$ be an integer number greater than 1 . Regarding the subsets $\mathrm{T}=\mathrm{T}_{v}$ and $\mathrm{V}=\mathrm{V}_{v}$ of $\mathrm{GL}_{n}(\mathbb{k})$, the following assertions are true.

(i) TVT is contained in $\mathrm{T}$, whose intersection with $Z\left(\mathrm{GL}_{n}(\mathrm{k})\right)$ is empty; and

(ii) if $\mathrm{T}$ and $\mathrm{V}$ are nonempty, then $\mathrm{TV}$ and $\mathrm{VT}$ are multiplicative semigroups disjoint from $Z\left(\mathrm{GL}_{n}(\mathrm{k})\right)$ and $\mathrm{VTV}$ is also disjoint from $Z\left(\mathrm{GL}_{n}(\mathrm{k})\right)$.

Proof: The result is vacuously true if one among $\mathrm{T}$ and $\mathrm{V}$ is empty. Otherwise, we fix arbitrary elements $P$ and $Q$ in $\mathrm{T}$ and $A=\operatorname{diag}\left(a_{1}, \ldots, a_{n}\right)$ in $\mathrm{V}$. Without loss of generality, we assume that $A$ belongs to $\mathrm{V}_{v}^{1}$, which particularly entails $a_{1} \neq a_{j}$, for each $j$ in $\{2, \ldots, n\}$ and so $A$ is non central. We write $v(P)$ and $v(Q)$ to denote the finite and constant valuation of the entries of $P$ and $Q$ respectively. For each $i$ and $\ell$ in $\{1, \ldots, n\}$,

$$
\begin{aligned}
v(P A Q(i, \ell)) & =v\left(\sum_{j=1}^{n} \sum_{k=1}^{n} P(i, j) A(j, k) Q(k, \ell)\right) \\
& =v\left(\sum_{j=1}^{n} a_{j} P(i, j) Q(j, \ell)\right) \\
& =v\left(a_{1}\right)+v(P)+v(Q),
\end{aligned}
$$

since, for each $j$ in $\{2, \ldots, n\}$,

$$
v\left(a_{j} P(i, j) Q(j, \ell)\right)=v\left(a_{j}\right)+v(P)+v(Q)>v\left(a_{1}\right)+v(P)+v(Q)
$$

and it thus follows that $P A Q$ belongs to $\mathrm{T}$. Consequently, $(\mathrm{TV})^{2}$ and $(\mathrm{VT})^{2}$ are respectively contained in $\mathrm{TV}$ and $\mathrm{VT}$ and so these are multiplicative semigroups. Since all the entries of an arbitrary member of T V, V T or V T V are nonzero, none of such sets shares elements with $Z\left(\mathrm{GL}_{n}(\mathrm{k})\right)$, which finishes this proof.

By a free pair, we understand a pair $\{g, h\}$ of elements of a group $G$ which freely generate a free subgroup. In order to exhibit free groups in the group of units of a matrix ring, we must combine lemma 2.1.1 with these well known facts recorded in the following

Proposition 2.1.2. If $\{g, h\}$ is a free pair in a group $G$, then $\{g h, h g\}$ is a free pair. If $z$ belongs to $\mathrm{Z}(G)$, then $\{z g, h\}$ also is a free pair.

Proof: Since $F=\langle g, h\rangle$ is freely generated by $g$ and $h$, we may consider the group homomorphism $\phi: F \rightarrow\langle g h, h g\rangle$ determined by the conditions

$$
\phi(g)=g h \text { and } \phi(h)=h g .
$$


Furthermore, for each member $w$ of $F$, we may also consider its length $\ell(w)$ and so the first assertion of the statement will be proved after we have shown that

$$
\ell(\phi(w))=2 \ell(w)
$$

Indeed, the equation above is manifest if $\ell(w) \leqslant 1$. If $\ell(w)=2$, then $\phi(w)$ is either one of the products

$$
(g h)(h g)^{-1},(g h)^{2},(g h)(h g),(h g)(g h),(h g)^{2} \text { and }(g h)^{-1}(h g)
$$

or one of their inverses. Whichever is the case, $\ell(\phi(w))=4$. We suppose that $n=\ell(w)$ is greater than 2 and work under the assumption that the result is valid for every element of $F$ with length up to $n-1$. Let $w_{1}, \ldots, w_{n-1}$ and $w_{n}$ be the members of $\left\{g, h, g^{-1}, h^{-1}\right\}$ such that $w=w_{1} \cdots w_{n-1} w_{n}$. From the induction hypothesis, it follows

$$
\ell\left(\phi\left(w_{1} \cdots w_{n-1}\right)\right)=2(n-1) \text { and } \ell\left(\phi\left(w_{n-1} w_{n}\right)\right)=4,
$$

which certainly ensures $\ell(\phi(w))=2 n$, as required.

For the proof of the second statement, we consider the group homomorphism $\varphi: F \rightarrow\langle z g, h\rangle$ determined by the conditions

$$
\varphi(g)=z g \text { and } \varphi(h)=h
$$

and the canonical homomorphism $\pi: G \rightarrow G / \mathrm{Z}(G)$. We have

$$
\pi(\varphi(g))=\pi(z g)=\pi(g) \text { and } \pi(\varphi(h))=\pi(h)
$$

hence, for each $w$ in $F, \pi(\varphi(w))=\pi(w)$, whence $\varphi(w)=1$ if and only if $\pi(w)=1$, which happens if and only if $w=1$. We conclude that $\varphi$ is an isomorphism and so this proof is complete.

Plenty of what we need so far has been established. We weave the results provided by lemma 2.1.1 and proposition 2.1.2 together and proceed to exhibit free groups contained in the group of units of a matrix ring over a field, with a few assumptions on some matrices and their images under non archimedean valuations.

Lemma 2.1.3. Let $v$ be a non archimedean valuation of a field $\mathbb{k}$ and $n$ be an integer number greater than 1 . Let $A, B, P$ and $Q$ be members of $\mathrm{GL}_{n}(\mathbb{k})$ such that

(i) $A, B$ and their inverses belong to $\mathrm{V}=\mathrm{V}_{v}$;

(ii) $P, Q$ and their inverses belong to $\mathrm{T}=\mathrm{T}_{v}$; and 
(iii) either $Q P=P Q=I$ or both $Q P$ and its inverse belong to $\mathrm{T}=\mathrm{T}_{v}$,

then $\{A P B Q, P B Q A\}$ is a free pair.

Proof: We fix an arbitrary nonzero integer number $k$. Manifestly, $A^{k}$ and $B^{k}$ belong to $\mathrm{V}$. Moreover, in agreement with lemma 2.1.1, if $Q P=P Q=I$, then

$$
(P B Q)^{k}=P B^{k} Q \text { belongs to } \mathrm{TVT} \subseteq \mathrm{T}
$$

and, if $P Q \neq I$, then either

$$
(P B Q)^{k}=P(B Q P)^{k-1} B Q \text { belongs to } \mathrm{T}(\mathrm{VT})^{k-1} \mathrm{VT} \subseteq \mathrm{T},
$$

if $k$ is positive, or

$$
(P B Q)^{k}=Q^{-1}\left(B^{-1} P^{-1} Q^{-1}\right)^{-(k+1)} B^{-1} P^{-1} \text { belongs to } \mathrm{T}(\mathrm{VT})^{-(k+1)} \mathrm{VT} \subseteq \mathrm{T},
$$

if $k$ is negative.

We write $F$ to denote the free group freely generated by the elements $X$ and $Y$. Let $\phi: F \rightarrow\langle A, P B Q\rangle$ be the group homomorphism determined by the conditions

$$
\phi(X)=A \text { and } \phi(Y)=P B Q .
$$

In light of the previous paragraph, it is readily seen that the image of any nontrivial member of $F$ under $\phi$ belongs to one among the sets T, V, T V, V T, and V T V and, since none of them intersects $\mathrm{Z}\left(\mathrm{GL}_{n}(\mathrm{k})\right)$, we conclude that $\phi$ is an isomorphism. It thus follows that $\{A, P B Q\}$ is a free pair and, by virtue of proposition 2.1.2. $\{A P B Q, P B Q A\}$ is a free pair.

Lemma 2.1.3 begets some useful results that have been used among the techniques to produce free groups, such as ([22], theorem 1), which is diluted in matricial versions in ([18], theorem 2.10) and in the following

Theorem 2.1.4 ([21], theorem 1). Let $\mathbb{H}$ be a division quaternion algebra over a field $\mathrm{k}$ whose characteristic is distinct from 2 . Let $\mathbf{i}$ and $\mathbf{j}$ be quaternion generators of $\mathbb{H}$ with squares respectively equal to $a$ and $b$. If there exist $\alpha, \beta$ and $\gamma$ in $\mathbb{k}^{\times}$and $a$ non archimedean valuation $v$ of $\mathbb{k}(\mathbf{j})$ such that $v(a)=v(b)=v(\alpha)=v(\beta)=0$ and $v(1+\gamma \mathbf{j}) \neq v(1-\gamma \mathbf{j})$, then $1+\gamma \mathbf{j}$ and $(\alpha \mathbf{i}+\beta \mathbf{j})(1+\gamma \mathbf{j})(\alpha \mathbf{i}+\beta \mathbf{j})$ freely generate a free group within $\mathbb{H}^{\times}$.

Proof: We denote $\omega=\alpha \mathbf{i}+\beta \mathbf{j}$ and $\theta=1+\gamma \mathbf{j}$. Regarding the structure of $\mathbb{H}$ as a left $\mathbb{k}(\mathbf{j})$-vector space with basis $\{1, \mathbf{i}\}$, the matrix obtained from $\omega$ by right regular representation is given by

$$
P=\left[\begin{array}{cc}
\beta \mathbf{j} & \alpha \\
a \alpha & -\beta \mathbf{j}
\end{array}\right] \text {. }
$$


The matrix accordingly obtained from $\theta$ is

$$
A=\left[\begin{array}{cc}
1+\gamma \mathbf{j} & 0 \\
0 & 1-\gamma \mathbf{j}
\end{array}\right]
$$

Since $A$ and $A^{-1}$ belong to $\mathrm{V}_{v}$ and both $P$ and $P^{-1}$ belong to $\mathrm{T}_{v},\left\{A, P^{-1} A P\right\}$ is a free pair. Since $P^{2}$ is central, it follows from proposition 2.1 .2 that $A$ and $P A P=P^{2} P^{-1} A P$ comprise a free pair. Equivalently, $\{\theta, \omega \theta \omega\}$ is a free pair.

Although some of our arguments ahead evoke the existence of elements with non central squares, which is registered in proposition 1.4.3, free groups may be rather easily exhibited within normal subgroups which contain non central elements of order 2 over its center. Theorem 2.1.4 shall help us when we find ourselves in such conditions.

\subsection{Valuations and algebraic arguments}

The previous section, when providing very useful lemmas, illustrates how important non archimedean valuations are to the process of constructing free groups in division rings. In acknowledgment of their importance, we describe non archimedean valuations that fit in with the results presented above.

The following result is of foremost interest. It is, in the context of our material, a suitably tailored version of the main theorem in [38] and has essentially appeared in distinct forms, as we see in ([22], lemma 7) and ([19], lemmas 2.3 and 2.4). Since it plays a major role in our reasoning, we register it below.

Lemma 2.2.1. Let $f(X)$ be an irreducible polynomial whose coefficients belong to a non absolute field $\mathbb{k}$. Let $\mathcal{A}$ be the collection of all roots of $f(X)$ in a splitting field $L$ over $\mathbb{k}$. We assume that $\mathcal{A}$ contains only nonzero elements, and so $f(X) \neq X$. If we are given an arbitrary member $\alpha_{0}$ of $\mathcal{A}$ and a finite subset $S$ of $L^{\times}$, there then exist infinitely many non archimedean valuations $v$ of $L$ such that

(i) $v(S)=0$; and

(ii) there exists $\eta$ in $\mathbb{k} \backslash\left\{\alpha_{0}\right\}$ such that $v(\eta)=0$, $v\left(\alpha_{0}-\eta\right)>0$ and, for each $\alpha$ in $\mathcal{A} \backslash\left\{\alpha_{0}\right\}, v(\alpha-\eta)=0$.

Proof: This proof follows the way that has been paved in [38]. Let $\mathcal{B}$ be a transcendence basis of $\mathbb{k}$ over its prime subfield $\mathbb{P}$. We shall give special attention to a subring $R$ of $\mathrm{k}$ and its field of fractions $Q$. They are determined depending on whether $\mathcal{B}$ is empty or not. In the first alternative, as a non absolute field, $k$ has 
characteristic zero and $\mathbb{P}$ may be treated as $\mathbb{Q}$, so we set $R=\mathbb{Z}$. In the second alternative, we fix an element $T$ in $\mathcal{B}$ and set $R=\mathbb{P}(\mathcal{B} \backslash\{T\})[T]$. Whichever is the case, $R$ is a principal ideal domain with infinitely many non associate prime elements and $\mathrm{k}$ is algebraic over $Q$. It is clear that $\mathcal{A}$ is contained in the collection $\widehat{\mathcal{A}}$ of all the roots of $\operatorname{irr}\left(\alpha_{0}, Q\right)$ and, since

$$
L_{0}=Q(S \cup \widehat{\mathcal{A}})
$$

is finite over $Q$, the integral closure $\mathcal{O}$ of $R$ in $L_{0}$ is a Dedekind domain with infinitely many prime ideals.

Throughout our discussion, we keep track of a particular non constant polynomial, which we describe in this paragraph. There certainly exists $\delta$ in $R \backslash\{0\}$ such that

$$
g(X)=\operatorname{irr}\left(\delta \alpha_{0}, Q\right)
$$

belongs to $R[X]$ and so

$$
\delta \widehat{A}=\{\delta \alpha \mid \alpha \in \widehat{A}\},
$$

which is promptly seen to be the collection of all roots of $g(X)$, is contained in $\mathcal{O}$. We conclude not only that $g(0)$ is nonzero, for $\alpha_{0}$ is nonzero and $g(X)$ is irreducible over $Q$, but also that $g(X)$ is a power of

$$
\prod_{\alpha \in \widehat{\mathcal{A}}}(X-\delta \alpha)
$$

We claim that there exists a family $\left\{\mathfrak{p}_{i} \mid i \geqslant 1\right\}$ of prime ideals of $\mathcal{O}$ with pairwise distinct intersections with $R$ and subsets $\left\{\eta_{i} \mid i \geqslant 1\right\}$ and $\left\{p_{i} \mid i \geqslant 1\right\}$ of $R$ such that, for each positive integer number $i, p_{i}$ generates $\mathfrak{p}_{i} \cap R$ and $\delta \alpha_{0}-\eta_{i}$ belongs to $\mathfrak{p}_{i}$, whereas $\eta_{i}$ and the members of $\delta \widehat{\mathcal{A}}$ do not. We apply an induction argument, as follows.

By means of the norm map $\mathfrak{N}: L_{0} \rightarrow Q$, we may clarify that it is possible to choose $\eta_{1}$ in $R \backslash\left\{\delta \alpha_{0}\right\}$ so that $\mathfrak{N}\left(\delta \alpha_{0}-\eta_{1}\right)$ is not a unit in $R$, which entails that $\delta \alpha_{0}-\eta_{1}$ is nonzero and non unit in $\mathcal{O}$. Working with a fixed basis of $L_{0}$ over $Q$, we write $M$ to denote the matrix of the multiplication map of $L_{0}$ corresponding to $\delta \alpha_{0}$. It is readily seen that, for an arbitrary member $\lambda$ of $R$, the matrix of the multiplication map of $L_{0}$ corresponding to $\delta \alpha_{0}-\lambda$ is $M-\lambda I$, therefore

$$
\mathfrak{N}\left(\delta \alpha_{0}-\lambda\right)=\operatorname{det}(M-\lambda I)
$$

is, up to sign, the evaluation at $\lambda$ of the characteristic polynomial of $M$, which is a power of $g(X)$, and so is a power of $g(\lambda)$. More generally, $\mathfrak{N}(\delta \alpha-\lambda)$ is a power of $g(\lambda)$, for each $\alpha$ in $\widehat{\mathcal{A}}$. 
We choose $\eta_{1}$ with two provisos. Firstly, since $g(0)$ is nonzero and $R$ is a principal ideal domain with infinitely many non associate prime elements, $\eta_{1}$ must be coprime to $g(0)$. Secondly, $\eta_{1}$ may be chosen to be a product of sufficiently high powers of prime elements of $R$ so that $g\left(\eta_{1}\right)$ is not a unit in $R$. More specifically, if $Q=\mathbb{Q}, \eta_{1}$ is chosen in $R=\mathbb{Z}$ so that $1+|\delta \alpha|<\left|\eta_{1}\right|$, for each $\alpha$ in $\widehat{\mathcal{A}}$, therefore

$$
1<\prod_{\alpha \in \widehat{\mathcal{A}}}\left(\left|\eta_{1}\right|-|\delta \alpha|\right) \leqslant \prod_{\alpha \in \widehat{\mathcal{A}}}\left|\eta_{1}-\delta \alpha\right|=\left|\prod_{\alpha \in \widehat{\mathcal{A}}}\left(\eta_{1}-\delta \alpha\right)\right|
$$

and so $\left|g\left(\eta_{1}\right)\right|>1$; otherwise, $Q$ has positive characteristic and $\eta_{1}$ is chosen in $R=\mathbb{P}(\mathcal{B} \backslash\{T\})[T]$ so that $\eta_{1}-\delta \alpha$ has positive degree in $T$, for each $\alpha$ in $\widehat{\mathcal{A}}$, hence the degree of

$$
\prod_{\alpha \in \widehat{\mathcal{A}}}\left(\eta_{1}-\delta \alpha\right)
$$

in $T$ is also positive, whence the same conclusion holds for $g\left(\eta_{1}\right)$.

In light of the previous paragraph, $\delta \alpha-\eta_{1}$ is nonzero and non unit, for each $\alpha$ in $\widehat{\mathcal{A}}$. In addition, $g(0)$ and $\eta_{1}$ are coprime, hence

$$
R=g(0) R+\eta_{1} R \subseteq(\delta \alpha) \mathcal{O}+\eta_{1} \mathcal{O}
$$

whence

$$
(\delta \alpha) \mathcal{O}+\eta_{1} \mathcal{O}=\mathcal{O}
$$

for each $\alpha$ in $\widehat{\mathcal{A}}$. We fix a prime ideal $\mathfrak{p}_{1}$ of $\mathcal{O}$ which contains $\delta \alpha_{0}-\eta_{1}$ and a generator $p_{1}$ of its intersection with $R$. Since each element of $\delta \widehat{\mathcal{A}}$ generates, together with $\eta_{1}$, the whole ring $\mathcal{O}$, we conclude that neither $\eta_{1}$ nor any member of $\delta \widehat{\mathcal{A}}$ belongs to $\mathfrak{p}_{1}$.

As an induction hypothesis, we assume that, for a positive integer number $n$, we have determined subsets $\left\{\eta_{1}, \ldots, \eta_{n}\right\}$ and $\left\{p_{1}, \ldots, p_{n}\right\}$ of $R$ and prime ideals $\mathfrak{p}_{1}, \ldots, \mathfrak{p}_{n}$ of $\mathcal{O}$ with the claimed properties. It is important to notice that each prime ideal of $\mathcal{O}$ lying above a prime divisor of $g(0)$ must divide $(\delta \alpha) \mathcal{O}$, for some $\alpha$ in $\widehat{\mathcal{A}}$, since $g(0) \mathcal{O}$ is a power of

$$
\left(\prod_{\alpha \in \widehat{\mathcal{A}}}(\delta \alpha)\right) \mathcal{O}=\prod_{\alpha \in \widehat{\mathcal{A}}}((\delta \alpha) \mathcal{O}) .
$$

Therefore none among $p_{1}, \ldots, p_{n}$ divides $g(0)$, for $\delta \widehat{\mathcal{A}}$ has empty intersection with $\mathfrak{p}_{i}=p_{i} R$, for each $i$ in $\{1, \ldots, n\}$. The choice of $\eta_{n+1}$ mirrors that of $\eta_{1}$, as we set it to be the product of sufficiently large positive powers of $p_{1}, \ldots, p_{n}$ so that $\delta \alpha_{0}-\eta_{n+1}$ is contained in a prime ideal $\mathfrak{p}_{n+1}$ of $\mathcal{O}$. It follows that $g(0)$ and $\eta_{n+1}$ are coprime, which entails that neither $\eta_{n+1}$ nor any member of $\delta \widehat{\mathcal{A}}$ belongs to $\mathfrak{p}_{n+1}$. We conclude 
that no generator of $\mathfrak{p}_{n+1} \cap R$ is associate to any element of $\left\{p_{1}, \ldots, p_{n}\right\}$ and fix $p_{n+1}$ in $R$ so that $p_{n+1} R=\mathfrak{p}_{n+1} \cap R$. The claim thus follows.

It is clear that there are infinitely many possibilities to chose a positive integer number $i$ such that $\mathfrak{p}_{i}$ is not a factor in the decomposition of any among the principal fractional ideals of $R$ generated by the elements of the finite set

$$
S \cup\{\delta\} \cup\left\{\alpha_{0}-\alpha \mid \alpha \in \mathcal{A} \backslash\left\{\alpha_{0}\right\}\right\}
$$

We consider the non archimedean valuation $v_{0}$ in $Q(S \cup \mathcal{A})$ obtained from the restriction of the $\mathfrak{p}_{i}$-adic valuation of $L_{0}$. In light of ([41], chapter 4 , theorem 1$), v_{0}$ admits an extension $v$ to $L$. It is readily seen that

$$
v(S)=0, v\left(\delta \alpha_{0}-\eta_{i}\right)>0 \text { and } v(\delta)=v\left(\alpha_{0}\right)=v\left(\eta_{i}\right)=v\left(\alpha_{0}-\alpha\right)=0,
$$

for each $\alpha$ in $\mathcal{A} \backslash\left\{\alpha_{0}\right\}$. We set

$$
\eta=\frac{\eta_{i}}{\delta}
$$

and observe that $v(\eta)=v\left(\eta_{i}\right)-v(\delta)=0$, hence $\eta \neq \alpha_{0}$,

$$
v\left(\alpha_{0}-\eta\right)=v\left(\delta \alpha_{0}-\eta_{i}\right)-v(\delta)=v\left(\delta \alpha_{0}-\eta_{i}\right)>0 .
$$

and, for each $\alpha$ in $\mathcal{A} \backslash\left\{\alpha_{0}\right\}$, since $\delta \alpha-\eta$ belongs to $\mathcal{O}$, we have

$$
v(\alpha-\eta)=v\left(\delta \alpha-\eta_{i}\right)-v(\delta) \geqslant 0
$$

therefore

$$
0 \leqslant \min \left\{v\left(\alpha_{0}-\eta\right), v(\alpha-\eta)\right\} \leqslant v\left(\alpha_{0}-\alpha\right)=0,
$$

which entails $v(\alpha-\eta)=0$. This proof is complete.

The equipment we have just gathered up will apply to the process of constructing free pairs in matrix rings. As it is to be expected, such rings will stand as targets of homomorphisms, whereas those division rings we regard as candidates of containing free pairs shall be settled in the domain. We close this chapter with a useful tool. It is, in a certain sense, a cancellation law which will help us to lift free pairs by means of homomorphisms. It has been stated in ([9], lemma 5.3) in a more general context, with a matrix ring $\mathrm{M}_{n}(D(T))$ instead of $D(T)$.

Lemma 2.2.2. Let $D$ be a division ring with an uncountable center $\mathrm{k}$. Let $T$ be a commutative indeterminate over $D$. If $\{f(T), g(T)\}$ is a free pair contained in $D(T)^{\times}$, there exists $\lambda$ in $\mathbb{k}$ such that $\{f(\lambda), g(\lambda)\}$ is a free pair in $D^{\times}$. Moreover, if * is an involution in $D$, then, regarding the natural extension of * to $D(T)$, if $f(T)$ and $g(T)$ are symmetric, then $\lambda$ may be chosen so that $f(\lambda)$ and $g(\lambda)$ are symmetric. 
Proof: We assume that $f(T)=f_{\mathrm{n}}(T) f_{\mathrm{d}}(T)^{-1}$ and $g(T)=g_{\mathrm{n}}(T) g_{\mathrm{d}}(T)^{-1}$ freely generate a free subgroup of $D(T)^{\times}$, therefore $f_{\mathrm{n}}(T), f_{\mathrm{d}}(T), g_{\mathrm{n}}(T)$ and $g_{\mathrm{d}}(T)$ are nonzero members of $D[T]$. Let $F$ be the free group generated by the set $\{X, Y\}$ and $\phi: F \rightarrow\langle f(T), g(T)\rangle$ be the group homomorphism determined by the conditions

$$
\phi(X)=f(T) \text { and } \phi(Y)=g(T) .
$$

Let $W$ be a nontrivial member of $F$. We denote

$$
\phi(W)=h^{W}(T)=h_{\mathrm{n}}^{W}(T) h_{\mathrm{d}}^{W}(T)^{-1}
$$

therefore

$$
h_{W}(T)=h_{\mathrm{n}}^{W}(T)-h_{\mathrm{d}}^{W}(T)
$$

is a nonzero member of $D[T]$. For each $\alpha$ in $\mathbb{k}$, the kernel of the evaluation homomorphism $\varepsilon_{\alpha}$ from $D[T]$ onto $D$ which sends $T$ to $\alpha$ is the principal ideal

$$
\operatorname{ker} \varepsilon_{\alpha}=(T-\alpha) D[T]
$$

hence $f_{\mathrm{n}}(\alpha), f_{\mathrm{d}}(\alpha), g_{\mathrm{n}}(\alpha), g_{\mathrm{d}}(\alpha)$ and $h_{W}(\alpha)$ are nonzero for all but finitely many elements $\alpha$ of $\mathbb{k}$. Moreover, since $F$ is countable, the collection

$$
\bigcup_{W \in F}\left\{\alpha \in \mathbb{k} \mid h_{W}(\alpha)=0\right\}
$$

accordingly is countable. It follows that there exists $\lambda$ in $\mathbb{k}$ such that $f_{\mathrm{n}}(\lambda), f_{\mathrm{d}}(\lambda)$, $g_{\mathrm{n}}(\lambda), g_{\mathrm{d}}(\lambda)$ and $h_{W}(\lambda)$ are nonzero, for each nontrivial $W$ in $F$. This proves that $f(\lambda)$ and $g(\lambda)$ freely generate a free group within $D^{\times}$.

Since $\left[\mathbb{k}: \mathbb{k}_{*}\right] \leqslant 2, \mathbb{k}_{*}$ is uncountable and we may assume that $\lambda$ is symmetric. If $f(T)=\left(\sum \omega_{i} T^{i}\right)\left(\sum \theta_{i} T^{i}\right)^{-1}$ is symmetric with respect to the extension of $*$ to $D(T)$, then

$$
\left(\sum \theta_{i}^{*} T^{i}\right)^{-1}\left(\sum \omega_{i}^{*} T^{i}\right)=f(T)^{*}=f(T)=\left(\sum \omega_{i} T^{i}\right)\left(\sum \theta_{i} T^{i}\right)^{-1}
$$

and so

$$
f(\lambda)^{*}=\left(\left(\sum \omega_{i} \lambda^{i}\right)\left(\sum \theta_{i} \lambda^{i}\right)^{-1}\right)^{*}=\left(\sum \theta_{i}^{*} \lambda^{i}\right)^{-1}\left(\sum \omega_{i}^{*} \lambda^{i}\right)=f(\lambda) .
$$

The same argument shows that $g(\lambda)$ is symmetric and completes this proof. 



\section{Chapter 3}

\section{Free symmetric pairs in normal subgroups}

\subsection{Existence of non central symmetric elements}

Let $D$ be a division ring with center $\mathrm{k}$, a field whose characteristic is distinct from 2. By a free symmetric pair, we understand a free pair in $D^{\times}$which is comprised by symmetric elements with respect to a given involution in $D$. Free unitary pairs are defined in the analogous manner. We assume that $D$ is finite dimensional over $k$ and is endowed with an involution of the first kind. As it is the case, for instance, in [18], in ([12], theorem 5) and in several results we are to present, the reasoning to prove the existence of free symmetric pairs in a non central normal subgroup $N$ of $D^{\times}$usually keeps track of a symmetric non central element, say $\omega$, assumed to exist in $N$. Such approach is justified by the fact that, taking into account a long process of embedding $D$ into several larger structures where we perform operations with $\omega$, such element acts like an anchor so that, in the end, we are able to settle in $N$ those free symmetric pairs detected in broader environments. We are thus motivated to introduce the following

Theorem 3.1.1. Let $D$ be a noncommutative division ring of finite dimension over its center $\mathbb{k}$, a field whose characteristic is distinct from 2 . Let $*$ be a $\mathbb{k}$-involution in $D$ and $N$ be a non central normal subgroup of $D^{\times}$. Unless $D$ is a quaternion algebra endowed with the symplectic involution, $N$ contains a non central symmetric element.

Proof: In light of proposition 1.4.3, we may work with a fixed element $\omega$ in $N$ whose square is non central. Let $K$ be a maximal subfield of $D$ containing $\omega$. The degree of $K$ over $\mathbb{k}$ (namely, the degree of $D$ ) and the degree of $\mathbb{k}(\omega)$ over $\mathbb{k}$ shall 
be respectively denoted by $n$ and $r$. By virtue of proposition $1.4 .2, K$ is separable over $\mathrm{k}$ and we may consider its Galois closure $L$, which thus contains the $r$ (distinct) roots $\omega_{1}, \omega_{2}, \ldots, \omega_{r}$ of $\operatorname{irr}(\omega, \mathbb{k})$. In agreement with the primitive element theorem, there exists $\alpha$ in $L$ such that $L=\mathbb{k}(\alpha)$.

We consider the $L$-isomorphism $\phi: D_{\alpha} \rightarrow \mathrm{M}_{n}(L)$. Regarding the extension of * to $D_{\alpha}$, for each $A$ in $\mathrm{M}_{n}(L)$, we write $A^{*}$ to denote

$$
\phi\left(\phi^{-1}(A)^{*}\right)
$$

and it is clear that the rule that assigns $A^{*}$ to $A$ is an $L$-involution in $\mathrm{M}_{n}(L)$. Regarding the evaluation map $\pi_{\alpha}: D_{\alpha}(T) \rightarrow D_{\alpha}$, if we write $\pi_{\alpha}$ instead of $\phi \pi_{\alpha}$, it may be considered as a surjective homomorphism from $D_{\alpha}(T)$ onto $\mathrm{M}_{n}(L)$ which thus obeys

$$
\pi_{\alpha}\left(f(T)^{*}\right)=\pi_{\alpha}(f(T))^{*}=f(\alpha)^{*}
$$

for each $f(T)$ in $D_{\alpha}(T)$.

Allover this proof, we keep track of the matrix

$$
\Omega=\pi_{\alpha}(\omega)
$$

Since $\pi_{\alpha}$ embeds $D$ into $\mathrm{M}_{n}(L), \omega$ and $\Omega$ have the same minimal polynomial (which is separable) over $\mathrm{k}$, hence $\Omega$ is diagonalizable in $\mathrm{M}_{n}(L)$ and its eigenvalues are roots of $\operatorname{irr}(\omega, \mathbb{k})$. In other words, there exists $W$ in $\operatorname{GL}_{n}(L)$ such that $\Omega^{W}$ is diagonal and the entries along its main diagonal belong to the set $\left\{\omega_{1}, \omega_{2}, \ldots, \omega_{n}\right\}$. As well as $\Omega^{2}$, the square of the diagonal matrix $\Omega^{W}$ is non central, hence there exist distinct roots $\omega^{\prime}$ and $\omega^{\prime \prime}$ of $\operatorname{irr}(\omega, \mathbb{k})$ which are eigenvalues of $\Omega$ whose squares are unequal. Of course, we may assume $\omega^{\prime}=\omega_{1}$ and $\omega^{\prime \prime}=\omega_{2}$.

In agreement with proposition 1.3 .3 , there exist matrices $U$ and $V$ in $\operatorname{GL}_{n}(L)$ such that, for all $A$ in $\mathrm{M}_{n}(L)$, the rule that assigns

$$
A^{\bullet}=U^{-1} A^{\top} U
$$

to $A$ is an $L$-involution in $\mathrm{M}_{n}(L)$ and

$$
A^{* V}=A^{V \bullet} \text { or, equivalently, } A^{V^{-1} *}=A^{\bullet V^{-1}} .
$$

We observe that

$$
\Omega^{W \bullet}=U^{-1}\left(\Omega^{W}\right)^{\top} U=\Omega^{W U}
$$

and split the arguments ahead in two cases, considering whether $U$ is diagonal or not. 


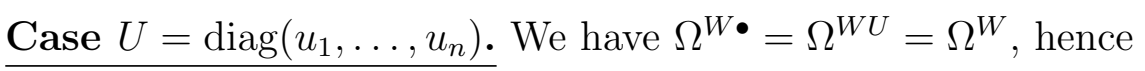

$$
\Omega^{W V^{-1}} \Omega^{W V^{-1} *}=\Omega^{W V^{-1}} \Omega^{W \bullet V^{-1}}=\left(\Omega^{W} \Omega^{W \bullet}\right)^{V^{-1}}=\left(\left(\Omega^{W}\right)^{2}\right)^{V^{-1}}=\left(\Omega^{2}\right)^{W V^{-1}}
$$

whence $\Omega^{W V^{-1}} \Omega^{W V^{-1} *}$ is non central. There exists an invertible element $f(T)$ in $D_{\alpha}(T)$ such that

$$
W V^{-1}=f(\alpha)
$$

therefore

$$
\pi_{\alpha}\left(\omega^{f(T)} \omega^{f(T) *}\right)=\Omega^{W V^{-1}} \Omega^{W V^{-1} *} .
$$

It follows that $\omega^{f(T)} \omega^{f(T) *}$ is non central and so there exists $g(T)$ in $D_{\alpha}(T)$ such that

$$
\omega^{f(T)} \omega^{f(T) *} g(T)-g(T) \omega^{f(T)} \omega^{f(T) *}
$$

is nonzero. Since $\mathbb{k}$ necessarily is an infinite field, we have enough room to choose $\lambda$ in $\mathrm{k}$ so that

$$
\omega^{f(\lambda)} \omega^{f(\lambda) *} g(\lambda)-g(\lambda) \omega^{f(\lambda)} \omega^{f(\lambda) *} \neq 0 .
$$

It is clear that $\omega^{f(\lambda)} \omega^{f(\lambda) *}$ is a non central symmetric element of $N$.

Case $U$ is not diagonal. In accordance with proposition 1.3 .3 , there exists an integer number $m$ such that $n=2 m$ and $U$ is written in block form

$$
U=\left[\begin{array}{cc}
0 & -\mathrm{I}_{m} \\
\mathrm{I}_{m} & 0
\end{array}\right] .
$$

Furthermore, $m$ necessarily is greater than 1 , since, otherwise, $D$ would be a quaternion algebra and $*$ would be the symplectic involution. There exist diagonal matrices $M_{1}$ and $M_{2}$ in $\mathrm{GL}_{m-2}(L)$ such that

$$
\Omega^{W}=\operatorname{diag}\left(a_{1}, a_{2}\right) \oplus M_{1} \oplus \operatorname{diag}\left(b_{1}, b_{2}\right) \oplus M_{2}
$$

and so

$$
\Omega^{W} \Omega^{W \bullet}=\operatorname{diag}\left(a_{1} b_{1}, a_{2} b_{2}\right) \oplus\left(M_{1} M_{2}\right) \oplus \operatorname{diag}\left(a_{1} b_{1}, a_{2} b_{2}\right) \oplus\left(M_{1} M_{2}\right),
$$

where $a_{1}, a_{2}, b_{1}$ and $b_{2}$ belong to $\left\{\omega_{1}, \omega_{2}, \ldots, \omega_{r}\right\}$. In order to choose $W$, the first property we demand is

$$
\left(a_{1}, a_{2}\right)=\left(\omega_{1}, \omega_{2}\right) .
$$

If we are given eigenvalues $\omega_{i}$ and $\omega_{j}$ of $\Omega$, where $i$ and $j$ are (not necessarily distinct) members of $\{1, \ldots, r\}$, then one among the inequalities

$$
\omega_{1} \omega_{i} \neq \omega_{2} \omega_{j} \text { and } \omega_{2} \omega_{i} \neq \omega_{1} \omega_{j}
$$


must hold, for $\omega_{1}^{2} \neq \omega_{2}^{2}$. Reversing the roles of $i$ and $j$, if necessary, the second property we demand is

$$
\left(b_{1}, b_{2}\right)=\left(\omega_{i}, \omega_{j}\right),
$$

therefore $a_{1} b_{1} \neq a_{2} b_{2}$. Whichever is the case,

$$
\Omega^{W V^{-1}} \Omega^{W V^{-1} *}=\left(\Omega^{W} \Omega^{W \bullet}\right)^{V^{-1}}
$$

is non central. In agreement with the reasoning to the proof of the previous case, this is sufficient to conclude that $N$ contains a non central symmetric element.

Aside from what we discussed in the opening of this section, theorem 3.1 .1 is also important from two more points of view. Firstly, its proof anticipates several arguments we shall apply to demonstrate theorem 3.2.2, which justifies our detailed explanation above. Essentially, it describes the relationship among $D, D_{\alpha}$ and $D(T)$; how we lift objects from $D_{\alpha}$ to $D(T)$; and, with aid of lemma 2.2.2, how we settle objects from $D(T)$ in $N$. Moreover, as we shall testify in the beginning of the proof of theorem 3.2 .2 (where $D$ is assumed to be finite dimensional and $\mathbb{k}$ is assumed to be uncountable), it may be fused with lemma 3.2.1 in order to avoid the fact that $N / \mathrm{Z}(N)$ is not torsion, which may be obtained from ([25], theorem 2) or from Tits' alternative [44] under disguise in ([11], theorem 2.1).

Remark 3.1.2. Regarding a division quaternion algebra $\mathbb{H}$ endowed with an orthogonal involution $*$, in comparison to theorem 3.1.1, a simpler proof is available. Indeed, we fix a normal subgroup $N$ of $\mathbb{H}^{\times}$with no non central symmetric elements. By virtue of the discussion preceding proposition 1.4.1, $N$ is invariant under $*$ and so the map which assigns $\omega \omega^{*}$ to each $\omega$ in $N$ is readily seen to be a group homomorphism from $N$ to $\mathbb{k}$ whose kernel is comprised by the unitary elements contained in $N$. Since the image of such homomorphism is abelian, the commutator subgroup $[N, N]$ is contained in the group of unitary elements of $\mathbb{H}^{\times}$, which is known to be commutative. As a characteristic subgroup of $N,[N, N]$ is normal in $\mathbb{H}^{\times}$and so, by virtue of the corollary 1.1.5, $[N, N]$ must be contained in $\mathrm{k}$. It means that

$$
\frac{N \mathbb{k}^{\times}}{\mathbb{k}^{\times}} \cong \frac{N}{N \cap \mathbb{k}^{\times}}
$$

is a abelian subgroup of $\mathbb{H}^{\times} / \mathbb{k}^{\times}$. In light of theorem 1.1.4, we conclude that $N$ is central.

\subsection{Main results}

We begin with a generalization of ([20], theorem 3.8) which is adapted to the context of involutions. As it was foreshadowed in the end of the section 2.1, we 
present the fundamental

Lemma 3.2.1. Let $D$ be a noncommutative division ring with center $\mathbb{k}$, a field whose characteristic is distinct from 2 , and endowed with an involution *. A normal subgroup $N$ of $D^{\times}$which contains a non central symmetric element $\omega_{0}$ with central square necessarily contains a free symmetric pair. In addition, if $*$ is of the second kind, then $N$ also contains a free unitary pair.

Proof: In order to use theorem 2.1.4. $\omega_{0}$ must be accommodated within a certain division quaternion algebra $\mathbb{H}$ contained in $D$, with symmetric generators $\mathbf{i}$ and $\mathbf{j}$ whose accordingly symmetric squares are respectively denoted by $a$ and $b$. The center of $\mathbb{H}$ shall be $K=\mathrm{k}_{\mathrm{k}}(a, b)$. In what follows, we work to give meaning to the notation we have just introduced.

It is readily seen that $\omega_{0}$ and $-\omega_{0}$ share the same minimal polynomial over $k$ and so, in agreement with theorem 1.1.3, they are conjugate in $D$, hence there exists $\theta_{0}$ in $D^{\times}$such that

$$
\omega_{0} \theta_{0}=-\theta_{0} \omega_{0}
$$

Consequently, equations

$$
\theta_{0}^{*} \omega_{0}=-\omega_{0} \theta_{0}^{*} \text { and }\left(\theta_{0}-\theta_{0}^{*}\right) \omega_{0}=-\omega_{0}\left(\theta_{0}-\theta_{0}^{*}\right)
$$

hold and both $\theta_{0}^{2}$ and $\left(\theta_{0}-\theta_{0}^{*}\right)^{2}$ commute with $\omega_{0}$. We set $\mathbf{i}=\omega_{0}$ and either $\mathbf{j}=\theta_{0}$, if $\theta_{0}$ is symmetric, or $\mathbf{j}=\omega_{0}{ }^{-1}\left(\theta_{0}-\theta_{0}^{*}\right)$ otherwise. It follows that $\mathbf{i}$ and $\mathbf{j}$ are both symmetric and so $\mathbb{H}$ is a division quaternion algebra over $K$ invariant under $*$ and whose normal subgroup

$$
N_{0}=N \cap \mathbb{H}
$$

contains the non central symmetric element i. We proceed to show that $N_{0}$ contains a free symmetric pair.

We claim that there exist $\alpha$ and $\beta$ in $K^{\times}$such that $\omega=\alpha \mathbf{i}+\beta \mathbf{j}$ is a symmetric member of $N_{0}$. Indeed, for each $\lambda$ in $K, 1+\lambda \mathbf{k}$ is nonzero and

$$
(1+\lambda \mathbf{k})^{-1} \mathbf{i}(1+\lambda \mathbf{k})=\frac{1-a b \lambda^{2}}{1+a b \lambda^{2}} \mathbf{i}+\frac{2 a \lambda}{1+a b \lambda^{2}} \mathbf{j}
$$

is a member of $N_{0}$. Since $K$ is a non absolute field and $\left[K: K_{*}\right] \leqslant 2, \lambda$ may be chosen in $K_{*}^{\times}$so that we avoid any of the roots of the polynomial $1+a b X^{2}$. The claim follows.

By virtue of lemma 2.2.1, there exists a non archimedean valuation $v$ of $K(\mathbf{j})$ and a member $\eta$ of $K_{*}^{\times}$such that

$$
v(a)=v(b)=v(\alpha)=v(\beta)=v(\eta)=v(\mathbf{j}+\eta)=0 \text { and } v(\mathbf{j}-\eta)>0 .
$$


We write $\gamma=\eta^{-1}$, hence

$$
v(1+\gamma \mathbf{j})=0 \text { and } v(1-\gamma \mathbf{j})>0
$$

As well as $\omega=\alpha \mathbf{i}+\beta \mathbf{j}$,

$$
\theta=\frac{(1+\gamma \mathbf{j})^{2}}{1-b \gamma^{2}}=(1-\gamma \mathbf{j})^{-1} \mathbf{i}^{-1}(1-\gamma \mathbf{j}) \mathbf{i}
$$

is a member of $N_{0}$. By virtue of theorem 2.1.4. $\{\omega, \omega \theta \omega\}$ is a free symmetric pair in $N_{0}$, as required.

We work now under the assumption that $*$ is an involution of the second kind. Regarding the notation established above and the structure of $\mathbb{H}$ as a left $K(\mathbf{j})$-vector space with basis $\{1, \mathbf{i}\}$, the matrix obtained from $\omega$ by right regular representation is given by

$$
P=\left[\begin{array}{cc}
\beta \mathbf{j} & \alpha \\
\alpha \alpha & -\beta \mathbf{j}
\end{array}\right] .
$$

We may fix a skew-symmetric element $\lambda$ in $K \backslash K_{*}$ in the same manner we did in proposition 1.3.2. With the aid the lemma 2.2.1, we choose the non archimedean valuation $v$ of $K(\mathbf{j})$ and the member $\eta$ of $K_{*}^{\times}$this time so that the images of the entries of $P$ and $P^{-1}$ under $v$ are zero, $v(\eta+\lambda \mathbf{j})=0$ and $v(\eta-\lambda \mathbf{j})>0$ and denote

$$
\theta=\eta+\lambda \mathbf{j} \text { and } \mu=\eta^{2}-b \lambda^{2}
$$

It follows that

$$
\theta^{*}=\mu \theta^{-1}
$$

and not only the matrix $A$ obtained from $\theta$ by right regular representation, but also its inverse belongs to $\mathrm{V}_{v}$, whereas $P$ and its inverse belong to $\mathrm{T}_{v}$. By virtue of the lemma 2.1.3 $A^{-1} P^{-1} A P$ and $P^{-1} A P A^{-1}$ freely generate a free group contained in $\mathrm{GL}_{2}(K(\mathbf{j}))$. With greater reason, $\left\{\theta^{-1} \omega^{-1} \theta \omega, \omega^{-1} \theta \omega \theta^{-1}\right\}$ is a free unitary pair in $N_{0}$, for $\omega^{*}=\omega=\xi \omega^{-1}$, where

$$
\xi=a \alpha^{2}+b \beta^{2}
$$

and

$$
\begin{aligned}
\left(\theta^{-1} \omega^{-1} \theta \omega\right)^{*} & =\omega^{*} \theta^{*} \omega^{-*} \theta^{-*} \\
& =\left(\xi \omega^{-1}\right)\left(\mu \theta^{-1}\right)\left(\xi \omega^{-1}\right)^{-1}\left(\mu \theta^{-1}\right)^{-1} \\
& =\omega^{-1} \theta^{-1} \omega \theta \\
& =\left(\theta^{-1} \omega^{-1} \theta \omega\right)^{-1}
\end{aligned}
$$


and

$$
\begin{aligned}
\left(\omega^{-1} \theta \omega \theta^{-1}\right)^{*} & =\theta^{-*} \omega^{*} \theta^{*} \omega^{-*} \\
& =\left(\mu \theta^{-1}\right)^{-1}\left(\xi \omega^{-1}\right)\left(\mu \theta^{-1}\right)\left(\xi \omega^{-1}\right)^{-1} \\
& =\theta \omega^{-1} \theta^{-1} \omega \\
& =\left(\omega^{-1} \theta \omega \theta^{-1}\right)^{-1}
\end{aligned}
$$

which completes this proof.

In due time, we shall sharpen lemma 3.2.1 in what it concerns the existence of free symmetric pairs. For the moment, we have everything we need in order to prove our main theorems. We begin with

Theorem 3.2.2. Let $D$ be a noncommutative division ring of finite dimension over its center $\mathbb{k}$, an uncountable field whose characteristic is distinct from 2 . Let $*$ be a k-involution in $D$ and $N$ be a non central normal subgroup of $D^{\times}$. Unless $D$ is a quaternion algebra endowed with the symplectic involution, $N$ contains a free symmetric pair.

Proof: In light of theorem 3.1.1 and lemma 3.2.1, we may assume that there exists $\omega$ in $N$ such that, for each natural number $k, \omega^{2^{k}}$ and its inverse are non central. We reintroduce the notation used in the proof of theorem 3.1.1. The positive integer numbers $n$ and $r$ respectively are the index of $D$ and the degree of $\mathbb{k}(\omega)$ over $\mathbb{k}$, $L=\mathbb{k}(\alpha)$ is the Galois closure of a maximal subfield of $D$ which contains $\omega$ and so the collection $\left\{\omega_{1}, \ldots, \omega_{r}\right\}$ of all roots of $\operatorname{irr}(\omega, \mathbb{k})$ is contained in $L$. We consider the evaluation map $\pi_{\alpha}: D_{\alpha}(T) \rightarrow \mathrm{M}_{n}(L)$, which obeys

$$
\pi_{\alpha}\left(f(T)^{*}\right)=\pi_{\alpha}(f(T))^{*}=f(\alpha)^{*}
$$

for each $f(T)$ in $D_{\alpha}(T)$. We recall that, in agreement with proposition 1.3.3, there exist matrices $U$ and $V$ in $\mathrm{GL}_{n}(L)$ such that, for each $A$ in $\mathrm{M}_{n}(L)$, the rule that assigns

$$
A^{\bullet}=U^{-1} A^{\top} U
$$

to $A$ is an $L$-involution and

$$
A^{* V}=A^{V \bullet} \text { or, equivalently, } A^{V^{-1} *}=A^{\bullet V^{-1}} \text {. }
$$

Once more, we write

$$
\Omega=\pi_{\alpha}(\omega)
$$

and recall that it is a diagonalizable matrix in $\mathrm{M}_{n}(L)$. Under the condition that $\omega^{2^{k}}$ and its inverse are non central for every natural number $k$, we may assume, in an 
analogous manner as we did in theorem 3.1.1, that $\omega_{1}$ and $\omega_{2}$ are eigenvalues of $\Omega$ such that $\omega_{1}^{8}$ and $\omega_{2}^{8}$ are distinct. We examine the cases ahead, regarding whether $U$ is diagonal or not.

Case $U=\operatorname{diag}\left(u_{1}, \ldots, u_{n}\right)$. An element $W$ of $\mathrm{GL}_{n}(L)$ may be chosen so that there exists a diagonal matrix $M$ in $\mathrm{GL}_{n-2}(L)$ such that

$$
\Omega^{W}=\operatorname{diag}\left(\omega_{1}, \omega_{2}\right) \oplus M
$$

In order to use lemmas 2.1.3 and 2.2.1, we expect to work with a matrix all of whose entries are nonzero. It is enough, as we shall see ahead, if we obtain a $2 \times 2$ such matrix at the expense of $\operatorname{diag}\left(\omega_{1}, \omega_{2}\right)$.

Since $L$ is an infinite field, there exists $\beta$ in $L^{\times}$which does not annihilate, for each $k$ in $\{-2,-1,1,2\}$, the polynomial

$$
\left(X^{2}+u_{1} u_{2}\right)\left(\omega_{1}^{k} X^{2}+u_{1} u_{2} \omega_{2}^{k}\right)\left(\omega_{2}^{k} X^{2}+u_{1} u_{2} \omega_{1}^{k}\right)
$$

in $L[X]$. We denote $U_{0}=\operatorname{diag}\left(u_{1}, u_{2}\right)$ and

$$
E_{0}=\left[\begin{array}{cc}
u_{1} & -\beta \\
\beta & u_{2}
\end{array}\right]
$$

As a consequence of how $\beta$ was chosen in $L^{\times}$, we have

$$
\operatorname{det} E_{0}=\beta^{2}+u_{1} u_{2} \neq 0
$$

therefore we may consider, in $\mathrm{GL}_{2}(L)$, the matrix

$$
\begin{aligned}
P & =\operatorname{diag}\left(\omega_{1}, \omega_{2}\right)^{E_{0}} \\
& =\frac{1}{\beta^{2}+u_{1} u_{2}}\left[\begin{array}{cc}
\omega_{2} \beta^{2}+u_{1} u_{2} \omega_{1} & u_{2}\left(\omega_{2}-\omega_{1}\right) \beta \\
u_{1}\left(\omega_{2}-\omega_{1}\right) \beta & \omega_{1} \beta^{2}+u_{1} u_{2} \omega_{2}
\end{array}\right],
\end{aligned}
$$

and so, for each integer number $k$,

$$
\begin{aligned}
P^{k} & =\operatorname{diag}\left(\omega_{1}^{k}, \omega_{2}^{k}\right)^{E_{0}} \\
& =\frac{1}{\beta^{2}+u_{1} u_{2}}\left[\begin{array}{cc}
\omega_{2}^{k} \beta^{2}+u_{1} u_{2} \omega_{1}^{k} & u_{2}\left(\omega_{2}^{k}-\omega_{1}^{k}\right) \beta \\
u_{1}\left(\omega_{2}^{k}-\omega_{1}^{k}\right) \beta & \omega_{1}^{k} \beta^{2}+u_{1} u_{2} \omega_{2}^{k}
\end{array}\right] .
\end{aligned}
$$

Routinely calculation reveals the relation

$$
U_{0}^{-1} P^{\top} U_{0}=P
$$

Let $S$ be the collection of all the entries of $P, P^{2}$ and their inverses. The condition imposed to fix $\beta$ in $L^{\times}$and the assumption that $\omega_{1}^{8}$ and $\omega_{2}^{8}$ are distinct 
ensure that each element of $S$ is nonzero. By virtue of lemma 2.2.1, there exist a non archimedean valuation $v$ of $L$ and a member $\eta$ of $\mathbb{k}^{\times}$such that

$$
v(S)=0, v(\eta)=0 \text { and } v(\alpha+\eta)>0
$$

Not only $P$ and $P^{2}$, but also their inverses belong to $\mathrm{T}_{v}$ and

$$
A=\operatorname{diag}(\alpha+\eta, \eta)
$$

as well as its inverse, belongs to $\mathrm{V}_{v}$. In light of lemma 2.1.3,

$$
B_{1}=A^{-1} P A P=P^{A} P \text { and } B_{2}=U_{0}^{-1} B_{1}^{\top} U_{0}=P A P A^{-1}=P P^{A^{-1}}
$$

freely generate a free subgroup of $\mathrm{GL}_{2}(L)$.

We introduce the notation

$$
E=E_{0} \oplus \mathrm{I}_{n-2} \text { and } F=A \oplus \mathrm{I}_{n-2}
$$

therefore

$$
\Omega^{W E \bullet}=U^{-1}(P \oplus M)^{\top} U=\left(U_{0}^{-1} P^{\top} U_{0}\right) \oplus M=P \oplus M=\Omega^{W E},
$$

and $F^{\bullet}=F$. The argumentation in the previous paragraph leads to the conclusion that

$$
\Omega^{W E F} \Omega^{W E}=\left(P^{A} \oplus M\right)(P \oplus M)=B_{1} \oplus M^{2}
$$

and

$$
\Omega^{W E} \Omega^{W E F^{-1}}=(P \oplus M)\left(P^{A^{-1}} \oplus M\right)=B_{2} \oplus M^{2}
$$

freely generate a free subgroup of $\mathrm{GL}_{n}(L)$. Furthermore,

$$
\left(B_{1} \oplus M^{2}\right)^{\bullet}=U_{0}^{-1} B_{1}^{\top} U_{0} \oplus M^{2}=B_{2} \oplus M^{2},
$$

hence

$$
\left(\Omega^{W E F} \Omega^{W E}\right)^{V^{-1} *}=\left(\Omega^{W E F} \Omega^{W E}\right)^{\bullet V^{-1}}=\left(\Omega^{W E} \Omega^{W E F^{-1}}\right)^{V^{-1}} .
$$

In $D_{\alpha}(T)$, there certainly exist invertible elements $f(T)$ and $g(T)$ such that

$$
f(\alpha)=W E F V^{-1} \text { and } g(\alpha)=W E V^{-1},
$$

hence

$$
\left\{\omega^{f(T)} \omega^{g(T)},\left(\omega^{f(T)} \omega^{g(T)}\right)^{*}\right\}
$$

is a free pair contained in $D_{\alpha}(T)$. In light of proposition 2.1.2.

$$
\left\{\omega^{f(T)} \omega^{g(T)}\left(\omega^{f(T)} \omega^{g(T)}\right)^{*},\left(\omega^{f(T)} \omega^{g(T)}\right)^{*} \omega^{f(T)} \omega^{g(T)}\right\}
$$


is a free symmetric pair contained in $D_{\alpha}(T)$. By virtue of lemma 2.2.2, there exists $\lambda$ in $\mathbb{k}_{\text {such that }}$

$$
\left\{\omega^{f(\lambda)} \omega^{g(\lambda)}\left(\omega^{f(\lambda)} \omega^{g(\lambda)}\right)^{*},\left(\omega^{f(\lambda)} \omega^{g(\lambda)}\right)^{*} \omega^{f(\lambda)} \omega^{g(\lambda)}\right\}
$$

is a free symmetric pair within $D^{\times}$which is promptly seen to be contained in $N$.

Case $U$ is not diagonal. Let $m$ be the integer number greater than 1 such that $n=2 m$. As we have observed in the analogous case in the proof of theorem 3.1 .1 . there exist an element $W$ in $\mathrm{GL}_{n}(L)$ and a diagonal matrix $M$ in $\mathrm{GL}_{m-2}(L)$ such that

$$
\Omega^{W} \Omega^{W \bullet}=\operatorname{diag}\left(\theta_{1}, \theta_{2}\right) \oplus M \oplus \operatorname{diag}\left(\theta_{1}, \theta_{2}\right) \oplus M
$$

where $\theta_{1}$ and $\theta_{2}$ are products of roots of $\operatorname{irr}(\omega, \mathbb{k})$. This time, $W$ may be chosen so that $\theta_{1}^{4} \neq \theta_{2}^{4}$. Indeed, if we are given eigenvalues $\omega_{i}$ and $\omega_{j}$ of $\Omega$, where $i$ and $j$ are (not necessarily distinct) members of $\{1, \ldots, r\}$, then one among the inequalities

$$
\left(\omega_{1} \omega_{i}\right)^{4} \neq\left(\omega_{2} \omega_{j}\right)^{4} \text { and }\left(\omega_{2} \omega_{i}\right)^{4} \neq\left(\omega_{1} \omega_{j}\right)^{4}
$$

must hold, for $\omega_{1}^{8} \neq \omega_{2}^{8}$. Reversing the roles of $i$ and $j$, if necessary, $W$ may be chosen so that $\left(b_{1}, b_{2}\right)=\left(\omega_{i}, \omega_{j}\right)$. We thus have

$$
\theta_{1}^{k}+\theta_{2}^{k} \neq 0 \neq \theta_{2}^{k}-\theta_{1}^{k}
$$

for each $k$ in $\{-2,-1,1,2\}$.

This time, we set

$$
E_{0}=\left[\begin{array}{cc}
1 & -1 \\
1 & 1
\end{array}\right]
$$

and

$$
\begin{aligned}
P & =\operatorname{diag}\left(\theta_{1}, \theta_{2}\right)^{E_{0}} \\
& =\frac{1}{2}\left[\begin{array}{ll}
\theta_{1}+\theta_{2} & \theta_{2}-\theta_{1} \\
\theta_{2}-\theta_{1} & \theta_{1}+\theta_{2}
\end{array}\right],
\end{aligned}
$$

and so, for each integer number $k$,

$$
\begin{aligned}
P^{k} & =\operatorname{diag}\left(\theta_{1}^{k}, \theta_{2}^{k}\right)^{E_{0}} \\
& =\frac{1}{2}\left[\begin{array}{ll}
\theta_{1}^{k}+\theta_{2}^{k} & \theta_{2}^{k}-\theta_{1}^{k} \\
\theta_{2}^{k}-\theta_{1}^{k} & \theta_{1}^{k}+\theta_{2}^{k}
\end{array}\right] .
\end{aligned}
$$

Exactly as we have pointed out above, for each $k$ in $\{-2,-1,1,2\}$, all the entries of $P^{k}$ are nonzero. By virtue of lemma 2.2.1, there exists a non archimedean valuation $v$ of $L$ and a member $\eta$ of $\mathbb{k}^{\times}$such that $P, P^{2}$ and their inverses belong to $\mathrm{T}_{v}$ and

$$
A=\operatorname{diag}(\alpha+\eta, \eta)
$$


together with its inverse, belongs to $\mathrm{V}_{v}$. In light of lemma 2.1.3,

$$
B_{1}=A^{-1} P A P=P^{A} P \text { and } B_{2}=B_{1}^{\top}=P A P A^{-1}=P P^{A^{-1}}
$$

freely generate a free subgroup of $\mathrm{GL}_{2}(L)$.

We now set

$$
E=\left(E_{0} A\right) \oplus \mathrm{I}_{m-2} \oplus E_{0} \oplus \mathrm{I}_{m-2}
$$

and

$$
F=E_{0} \oplus \mathrm{I}_{m-2} \oplus\left(E_{0} A\right) \oplus \mathrm{I}_{m-2},
$$

therefore we have

$$
\begin{aligned}
& \Omega^{W E} \Omega^{W U E}=\left(\Omega^{W} \Omega^{W \bullet}\right)^{E}=P^{A} \oplus M \oplus P \oplus M \\
& \Omega^{W F} \Omega^{W U F}=\left(\Omega^{W} \Omega^{W \bullet}\right)^{F}=P \oplus M \oplus P^{A} \oplus M
\end{aligned}
$$

and also

$$
\begin{aligned}
\left(\Omega^{W E} \Omega^{W U E}\right)^{\bullet} & =P^{\top} \oplus M^{\top} \oplus\left(P^{A}\right)^{\top} \oplus M^{\top} \\
& =P \oplus M \oplus P^{A^{-1}} \oplus M
\end{aligned}
$$

and

$$
\begin{aligned}
\left(\Omega^{W F} \Omega^{W U F}\right)^{\bullet} & =\left(P^{A}\right)^{\top} \oplus M^{\top} \oplus P^{\top} \oplus M^{\top} \\
& =P^{A^{-1}} \oplus M \oplus P \oplus M
\end{aligned}
$$

It thus follows that

$$
\Omega^{W E} \Omega^{W U E}\left(\Omega^{W E} \Omega^{W U E}\right)^{\bullet}=B_{1} \oplus M^{2} \oplus B_{2} \oplus M^{2}
$$

and

$$
\Omega^{W F} \Omega^{W U F}\left(\Omega^{W F} \Omega^{W U F}\right)^{\bullet}=B_{2} \oplus M^{2} \oplus B_{1} \oplus M^{2}
$$

freely generate a free group in $\mathrm{GL}_{n}(L)$, in agreement with lemma 2.1.3. With greater reason,

$$
\Omega^{W E V^{-1}} \Omega^{W U E V^{-1}}\left(\Omega^{W E V^{-1}} \Omega^{W U E V^{-1}}\right)^{*}=\left(\Omega^{W E} \Omega^{W U E}\left(\Omega^{W E} \Omega^{W U E}\right)^{\bullet}\right)^{V^{-1}}
$$

and

$$
\Omega^{W F V^{-1}} \Omega^{W U F V^{-1}}\left(\Omega^{W F V^{-1}} \Omega^{W U F V^{-1}}\right)^{*}=\left(\Omega^{W F} \Omega^{W U F}\left(\Omega^{W F} \Omega^{W U F}\right)^{\bullet}\right)^{V^{-1}}
$$

freely generate a free subgroup of $\mathrm{GL}_{n}(L)$. This is sufficient, as it was in the previous case, to conclude that $N$ contains a free symmetric pair. 
As it was the case in lemma 3.2.1 and in some results in [12], we generally seek a quaternion algebra contained in a given division ring (sometimes even in a given division quaternion algebra) and which is invariant under the involution. We shall certainly explore such technique in several situations below, with the aid of our next main result, which partially generalizes ([9], lemma 2.6).

Theorem 3.2.3. Let $\mathrm{k}$ be a field of characteristic distinct from 2 and $\mathbb{H}$ be a division quaternion $\mathbb{k}$-algebra. Let $N$ be a non central normal subgroup of $\mathbb{H}^{\times}$. Regarding an involution * of the first kind in $\mathbb{H}$, the following assertions are true.

(i) If $*$ is orthogonal, then $N$ contains a free symmetric pair; and

(ii) if $*$ is symplectic, then $N$ contains a free unitary pair.

Proof: (i) In agreement with lemma 1.3.1, $\mathbb{H}$ admits symmetric quaternion generators $\mathbf{i}$ and $\mathbf{j}$ whose squares we respectively denote by $a$ and $b$. In light of remark 3.1 .2 , there exist $\alpha, \beta$ and $\gamma$ in $\mathbb{k}$ such that one among $\beta$ and $\gamma$ is nonzero and the non central symmetric element

$$
\omega=\alpha+\beta \mathbf{i}+\gamma \mathbf{j}
$$

belongs to $N$. If $\alpha=0$, the result follows from lemma 3.2.1. We may thus assume that $\alpha$ is nonzero and, unless we need to reverse the roles of $\mathbf{i}$ and $\mathbf{j}$, we may also assume that $\beta \neq 0$. More generally, the same argument and little computation allow us to assume that, for each $k$ in $\{-2,-1,1,2\}$, there exist $\alpha_{k}, \beta_{k}$ and $\gamma_{k}$ in $\mathbb{k}$ such that $\alpha_{k}$ and $\beta_{k}$ are nonzero and

$$
\omega^{k}=\alpha_{k}+\beta_{k} \mathbf{i}+\gamma_{k} \mathbf{j}
$$

Regarding the structure of $\mathbb{H}$ as a left $\mathbb{k}(\mathbf{j})$-vector space with basis $\{1$, $\mathbf{i}\}$, we write $P$ to denote the matrix obtained from $\omega$ by right regular representation. It thus follows that

$$
P^{k}=\left[\begin{array}{cc}
\alpha_{k}+\gamma_{k} \mathbf{j} & \beta_{k} \\
a \beta_{k} & \alpha_{k}-\gamma_{k} \mathbf{j}
\end{array}\right],
$$

for each $k$ in $\{-2,-1,1,2\}$. We set

$$
S=\left\{P^{k}(i, j) \mid i, j \in\{1,2\}, k \in\{-2,-1,1,2\}\right\}
$$

All the members of $S$ are nonzero and, by virtue of lemma 2.2.1, there exist a member $\eta$ of $\mathbb{k}$ and a non archimedean valuation $v$ of $\mathbb{k}(\mathbf{j})$ such that

$$
v(S)=0, v(\eta)=0, v(\eta-\mathbf{j})=0 \text { and } v(\eta+\mathbf{j})>0 .
$$


Furthermore, the matrix obtained from $\theta=\eta+\mathbf{j}$ by right regular representation is given by

$$
A=\left[\begin{array}{cc}
\eta+\mathbf{j} & 0 \\
0 & \eta-\mathbf{j}
\end{array}\right] .
$$

We observe that $P, P^{2}$ and their inverses belong to $\mathrm{T}_{v}$ and $A$, together with its inverse, belongs to $\mathrm{V}_{v}$. In light of lemma 2.1.3, $P^{A} P$ and $P P^{A^{-1}}$ freely generate a free subgroup of $\mathrm{GL}_{2}\left(\mathbb{k}_{\mathbf{k}}(\mathbf{j})\right)$. With greater reason, in agreement with proposition 2.1.2.

$$
\left(\omega^{\theta} \omega\right)\left(\omega^{\theta} \omega\right)^{*}=\left(\omega^{\theta} \omega\right)\left(\omega \omega^{\theta^{-1}}\right)
$$

and

$$
\left(\omega \omega^{\theta^{-1}}\right)\left(\omega \omega^{\theta^{-1}}\right)^{*}=\left(\omega \omega^{\theta^{-1}}\right)\left(\omega^{\theta} \omega\right)
$$

comprise a free symmetric pair contained in $N$.

(ii) We fix quaternion generators $\mathbf{i}$ and $\mathbf{j}$ of $\mathbb{H}$. Of course, $\mathbb{H}$ admits an orthogonal involution with respect to which $\mathbf{i}$ and $\mathbf{j}$ are both symmetric and, in light of the item (i), $N$ contains a free symmetric pair with respect to such involution. Consequently, there exist $\alpha, \beta$ and $\gamma$ in $\mathrm{k}$ such that $\alpha$ is nonzero,

$$
\omega=\alpha+\beta \mathbf{i}+\gamma \mathbf{j}
$$

belongs to $N$ and, unless we need to reverse the roles of $\mathbf{i}$ and $\mathbf{j}$, we may assume that $\beta$ is nonzero. Since $*$ is symplectic, $\mathbb{k}$ is precisely the collection of symmetric members of $\mathbb{H}$, hence there exists $\xi$ in $\mathbb{k}$ such that

$$
\begin{aligned}
\omega^{*} & =\alpha-\beta \mathbf{i}-\gamma \mathbf{j} \\
& =\xi \omega^{-1} .
\end{aligned}
$$

Regarding the structure of $\mathbb{H}$ as a left $\mathbb{k}(\mathbf{j})$-vector space with basis $\{1, \mathbf{i}\}$, the matrix obtained from $\omega$ by right regular representation is given by

$$
P=\left[\begin{array}{cc}
\alpha+\gamma \mathbf{j} & \beta \\
a \beta & \alpha-\gamma \mathbf{j}
\end{array}\right]
$$

and the one accordingly obtained from $\omega^{-1}$ is

$$
P^{-1}=\frac{1}{\xi}\left[\begin{array}{cc}
\alpha-\gamma \mathbf{j} & -\beta \\
-a \beta & \alpha+\gamma \mathbf{j}
\end{array}\right] .
$$

The members of the collection $S$ of all the entries of $P$ and $P^{-1}$ are nonzero and, by virtue of lemma 2.2.1, there exist an element $\eta$ of $\mathbb{k}$ and a non archimedean valuation

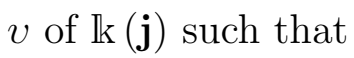

$$
v(S)=0, v(\eta)=0, v(\eta-\mathbf{j})=0 \text { and } v(\eta+\mathbf{j})>0 .
$$


The matrix obtained from $\theta=\eta+\mathbf{j}$ by right regular representation is given by

$$
A=\left[\begin{array}{cc}
\eta+\mathbf{j} & 0 \\
0 & \eta-\mathbf{j}
\end{array}\right]
$$

and there exists $\mu$ in $\mathbb{k}$ such that

$$
\begin{aligned}
\theta^{*} & =\eta-\mathbf{j} \\
& =\mu \theta^{-1} .
\end{aligned}
$$

We observe that $\left\{P, P^{-1}\right\}$ and $\left\{A, A^{-1}\right\}$ are respectively contained in $\mathrm{T}$ and in $\mathrm{V}$. In agreement with lemma 2.1.3,

$$
\left\{A^{-1} P^{-1} A P, P^{-1} A P A^{-1}\right\}
$$

is a free pair in $\mathrm{GL}_{2}(\mathbb{k})$. With greater reason,

$$
\left\{\theta^{-1} \omega^{-1} \theta \omega, \omega^{-1} \theta \omega \theta^{-1}\right\}
$$

is a free unitary pair in $N$, which is verified by means of the same computation we did in 3.2 .1 .

Remark 3.2.4. We observe that it is possible to take a shortcut to prove the item (ii) in theorem 3.2.3. Indeed, with respect to the symplectic involution in a division quaternion algebra $\mathbb{H}$, symmetric elements are central, therefore we proceed as in remark 3.1 .2 and conclude that the commutator subgroup $[N, N]$ of a non central normal subgroup $N$ of $\mathbb{H}^{\times}$is also a non central normal subgroup of $\mathbb{H}^{\times}$comprised by unitary elements. The result is thus achieved by virtue of ([11], theorem 2.4).

As a complement to theorem 3.2.3, we push the generalization of ([9], lemma 2.6) a bit further, in the following

Theorem 3.2.5. Let $\mathbb{H}$ be a division quaternion algebra with center $\mathbb{k}$, a field whose characteristic is distinct from 2. Let $N$ be a non central normal subgroup of $\mathbb{H}^{\times}$. If $\mathbb{H}$ is endowed with an involution * of the second kind, then $N$ contains a free symmetric pair.

Proof: In light of proposition 1.4.1, we may assume that $N$ is invariant under $*$. In accordance with proposition 1.3 .2 , $*$ is an involution of type II and, as it follows from lemma 1.3.1, $\mathbb{H}$ admits symmetric quaternion generators $\mathbf{i}$ and $\mathbf{j}$, whose accordingly symmetric squares we respectively denote by $a$ and $b$. We write $\mathbb{H}_{0}$ to denote the $\mathbb{k}_{*}$-subalgebra of $\mathbb{H}$ generated by $\mathbf{i}$ and $\mathbf{j}$. Manifestly, $\mathbb{H}_{0}$ is a division quaternion $\mathbb{k}_{*}$-algebra in which $*$ acts as an orthogonal involution. 
Firstly, we prove that $N$ contains a free symmetric pair under the assumption that it admits a non central symmetric member

$$
\begin{aligned}
\omega & =\alpha+\beta \mathbf{i}+\gamma \mathbf{j}+\delta \mathbf{k} \\
& =(\alpha+\gamma \mathbf{j})+(\beta-\delta \mathbf{j}) \mathbf{i},
\end{aligned}
$$

and so $\alpha, \beta$ and $\gamma$ belong to $\mathbb{k}_{*}$ and $\delta^{*}=-\delta$. If $\alpha=0$, the result immediately follows from lemma 3.2.1 and, if $\delta=0$, then $\omega$ is a non central member of the normal subgroup $N_{0}=N \cap \mathbb{H}_{0}$ of $\mathbb{H}_{0}^{\times}$, therefore, in agreement with theorem 3.2 .3 . $N_{0}$ and hence $N$ contains a free symmetric pair. We may thus assume that both $\alpha$ and $\delta$ are nonzero. We extend this argument for $\omega^{k}$, for each $k$ in $\{-2,-1,1,2\}$, concluding that there exist $\alpha_{k}, \beta_{k}$ and $\gamma_{k}$ in $\mathbb{k}_{*}$ and $\delta_{k}$ in $\mathbb{k}_{k}$ such that

$$
\begin{aligned}
\omega^{k} & =\alpha_{k}+\beta_{k} \mathbf{i}+\gamma_{k} \mathbf{j}+\delta_{k} \mathbf{k} \\
& =\left(\alpha_{k}+\gamma_{k} \mathbf{j}\right)+\left(\beta_{k}-\delta_{k} \mathbf{j}\right) \mathbf{i}
\end{aligned}
$$

and both $\alpha_{k}$ and $\delta_{k}$ are nonzero. Exactly as we have been proceeding, with the aid of proposition 2.1.2 and lemmas 2.1.3 and 2.2.1, we fix a member

$$
\theta=\eta+\mathbf{j}
$$

of $\mathbb{H}$, with $\eta$ in $\mathbb{k}_{*}$, and conclude that

$$
\left(\omega^{\theta} \omega\right)\left(\omega^{\theta} \omega\right)^{*}=\left(\omega^{\theta} \omega\right)\left(\omega \omega^{\theta^{-1}}\right)
$$

and

$$
\left(\omega \omega^{\theta^{-1}}\right)\left(\omega \omega^{\theta^{-1}}\right)^{*}=\left(\omega \omega^{\theta^{-1}}\right)\left(\omega^{\theta} \omega\right)
$$

comprise a free symmetric pair contained in $N$.

Our task now is to prove that $N$ contains a free symmetric pair even if we do not insist that it contains a non central symmetric member. Indeed, regarding the orthogonal involution in $\mathbb{H}$ under which both $\mathbf{i}$ and $\mathbf{j}$ are symmetric and in light of the item (i) of theorem 3.2.3, there exist $\alpha, \beta$ and $\gamma$ in $\mathbb{k}$ such that $\alpha$ and $\beta$ are nonzero and

$$
\begin{aligned}
\omega & =\alpha+\beta \mathbf{i}+\gamma \mathbf{j} \\
& =(\alpha+\gamma \mathbf{j})+\beta \mathbf{i} .
\end{aligned}
$$

The arguments in the previous paragraph allow us to assume that $\lambda=\omega \omega^{*}$ belongs to $\mathbb{k}_{*}$ and so

$$
\omega^{*}=\lambda \omega^{-1}
$$


Regarding the structure of $\mathbb{H}$ as a left $\mathbb{k}(\mathbf{j})$-vector space with basis $\{1, \mathbf{i}\}$, the matrix obtained from $\omega$ by right regular representation is given by

$$
P=\left[\begin{array}{cc}
\alpha+\gamma \mathbf{j} & \beta \\
a \beta & \alpha-\gamma \mathbf{j}
\end{array}\right]
$$

and the one accordingly obtained from $\omega^{-1}$ is

$$
P^{-1}=\frac{1}{\lambda}\left[\begin{array}{cc}
\alpha-\gamma \mathbf{j} & -\beta \\
-a \beta & \alpha+\gamma \mathbf{j}
\end{array}\right] .
$$

We write $S$ to denote the collection of all the entries of $P$ and $P^{-1}$, therefore all its members are nonzero. By virtue of lemma 2.2.1, there exist an element $\eta$ of $\mathbb{k}_{*}$ and a non archimedean valuation $v$ of $\mathbb{k}(\mathbf{j})$ such that

$$
v(S)=0, v(\eta)=0, v(\eta-\mathbf{j})=0 \text { and } v(\eta+\mathbf{j})>0 .
$$

The element $\theta=\eta+\mathbf{j}$ is symmetric and the matrix it determines by right regular representation is given by

$$
A=\left[\begin{array}{cc}
\eta+\mathbf{j} & 0 \\
0 & \eta-\mathbf{j}
\end{array}\right] .
$$

It is readily seen that $\left\{P, P^{-1}\right\}$ and $\left\{A, A^{-1}\right\}$ are respectively contained in $\mathrm{T}_{v}$ and in $\mathrm{V}_{v}$. In agreement with lemma 2.1.3,

$$
\left\{A^{-1} P^{-1} A P, P^{-1} A P A^{-1}\right\}
$$

is a free pair in $\mathrm{GL}_{2}(\mathbb{k})$. With greater reason,

$$
\left\{\theta^{-1} \omega^{-1} \theta \omega, \omega^{-1} \theta \omega \theta^{-1}\right\}
$$

is a free pair in $N$. Since

$$
\begin{aligned}
\left(\theta^{-1} \omega^{-1} \theta \omega\right)^{*} & =\omega^{*} \theta^{*} \omega^{-*} \theta^{-*} \\
& =\left(\lambda \omega^{-1}\right) \theta\left(\lambda \omega^{-1}\right)^{-1} \theta^{-1} \\
& =\omega^{-1} \theta \omega \theta^{-1}
\end{aligned}
$$

we conclude, with the aid of proposition 2.1 .2 , that

$$
\left(\theta^{-1} \omega^{-1} \theta \omega\right)\left(\omega^{-1} \theta \omega \theta^{-1}\right) \text { and }\left(\omega^{-1} \theta \omega \theta^{-1}\right)\left(\theta^{-1} \omega^{-1} \theta \omega\right)
$$

comprise a free symmetric pair contained in $N$, as required.

The proof of theorem 3.2 .5 does not follow the argument used in ([9], lemma 2.6 ), which consists in directly examine the multiplicative group of the division 
quaternion algebra $\mathbb{H}_{0}$ generated by symmetric quaternion generators of $\mathbb{H}$ over the field $k_{*}$. In order to work in such perspective, we would have to ensure that the normal subgroup $N_{0}$ of $\mathbb{H}_{0}^{\times}$given by the intersection of a non central normal subgroup $N$ of $\mathbb{H}^{\times}$with $\mathbb{H}_{0}$ were not contained in $\mathbb{k}_{*}$, which we suspect to be true, since non central normal subgroups of division rings may be understood as "big" sets, in accordance with ([43], theorem 4). In terms of theorem 3.2.5, the analogous difficulty we could not overcome in order to show that $N$ contains free unitary pairs is proving that $N$ has non central intersection with $\mathbb{H}_{0}$ when this is the division quaternion algebra generated over $\mathbb{k}_{*}$ by skew-symmetric quaternion generators of $\mathbb{H}$. We also believe this to be true. Fortunately, we are not totally clueless, since if one among the non central symmetric members of $N$ has central square, then lemma 3.2 .1 applies to show that there also exists a free unitary pair in $N$. We leave as a conjecture that this is true in the general case.

Since we are concerned with giving support to conjecture 4 mostly focused on free symmetric pairs, theorems 3.2.3 and 3.2.5 are, in their own right, obligatory part of this work, in the sense that division quaternion algebras comprise the family of the simplest division rings. Furthermore, in agreement with what we discussed above, several results may be derived from theorem them if we seek quaternion algebras (invariant under the given involution) within division rings. Form now on, all the results ahead may be regarded as consequences of the existence of free symmetric pairs in non central normal subgroups of division quaternion algebras.

Motivated by ([12], theorems 5 and 6 ), we attain the following

Theorem 3.2.6. Let $D$ be a noncommutative division ring which is algebraic over its center $\mathbb{k}$, a field whose characteristic is distinct from 2 . Let $*$ be a $\mathbb{k}$-involution in $D$ and $N$ be a non central normal subgroup of $D^{\times}$. If $N$ contains a non central element $\omega$ such that $\omega \omega^{*}$ is central, then $N$ contains a free symmetric or a free unitary pair.

Proof: Since $\omega \omega^{*}$ is central, there exists $\xi$ in $\mathbb{k}$ such that $\omega^{*}=\xi \omega^{-1}$. Since $\omega$ and $\omega^{*}$ share the same minimal polynomial over $k$, by virtue of theorem 1.1 .3 , there exists $\theta$ in $D^{\times}$such that

$$
\theta^{-1} \omega \theta=\omega^{*}=\xi \omega^{-1}
$$

Therefore $\theta^{2}$ centralizes $\omega$ and the following identities hold:

$$
\omega \theta=\theta \omega^{*}, \quad \omega^{*} \theta=\theta \omega, \quad \theta^{*} \omega^{*}=\omega \theta^{*} \text { and } \theta^{*} \omega=\omega^{*} \theta^{*}
$$

In light of lemma 3.2.1, we may assume that $\omega$ is unequal to $\omega^{*}$. Consequently, $\mathbf{i}=\omega-\omega^{*}$ is a nonzero skew-symmetric member of $D$ whose square we denote by $a$ 
and such that $\mathbf{i} \theta=-\theta \mathbf{i}$. We denote

$$
\text { either } \mathbf{j}=\theta \text {, if } \theta^{*}=\theta \text { or } \mathbf{j}=\theta-\theta^{*} \text {, otherwise, }
$$

and $b=\mathbf{j}^{2}$. Since $\mathbf{i} \theta^{*}=-\theta^{*} \mathbf{i}$, the identity $\mathbf{i} \mathbf{j}=-\mathbf{j} \mathbf{i}$ holds. Since $\omega \omega^{*}$ is central, we have

$$
\omega \omega^{*}=\omega^{*} \omega
$$

and so $c=\omega+\omega^{*}$ centralizes both $\mathbf{i}$ and $\mathbf{j}$. We conclude that the subring $\mathbb{H}$ of $D$ generated by $\mathbf{i}$ and $\mathbf{j}$ over $\mathbb{k}(a, b, c)$ is a division quaternion algebra contained in $D$ in which $*$ acts either as an orthogonal involution, if $\mathbf{j}$ is symmetric, or the symplectic involution, if $\mathbf{j}$ is skew-symmetric. The normal subgroup $N_{0}=N \cap \mathbb{H}$ of $\mathbb{H}^{\times}$is non central, for

$$
\omega=\frac{1}{2}(\mathbf{i}+c)
$$

belongs to $N_{0}$, but not to $\mathbb{k}(a, b, c)$. In light of theorem 3.2.3, $N_{0}$ and hence $N$ contains a free symmetric or a free unitary pair.

We recall the fact that the strategy for proving the existence of free symmetric pairs in a non central normal subgroup $N$ of a division ring $D$ with an involution * usually involves keeping track of a non central symmetric element assumed to exist in $N$. Theorem 3.2 .6 tells us that, if $D$ is algebraic over its center and $*$ is of the first kind and if $N$ fails to contain non central symmetric elements, then it must contain a free unitary pair. From the point of view of conjecturing that $N$ contains non cyclic free subgroups invariant under $*$, it is not far-fetched to demand that $N$ contains a non central symmetric member.

As a consequence of ([24], theorem 8), if all the members of a normal subgroup of a division ring have finite multiplicative order, then such subgroup is central. If we make such assumption on the multiplicative order of the symmetric elements of a division ring endowed with an involution, the conclusion remains true, in agreement with ([26], theorem 1). In what concerns a non central normal subgroup of a division ring finite dimensional over its center - whose characteristic is unequal to 2 - and endowed with an involution of the first kind, it is possible to prove the analogous statement, considering the collection of symmetric elements in the normal subgroup - which is nonempty, in light of theorem 3.1.1 - rather than all the symmetric members of the division ring. The piece we lack is given in the following

Theorem 3.2.7. Let $D$ be a noncommutative division ring of finite dimension over its center $\mathbb{k}$, a field whose characteristic $p$ is distinct from 2 . Let $*$ be a $\mathbb{k}$-involution in $D$ and $N$ be a non central normal subgroup of $D^{\times}$. If $N$ contains a non central symmetric element of finite multiplicative order, then $N$ contains a free symmetric pair. 
Proof: Let $\omega$ be a non central symmetric element of $N$ of finite multiplicative order $q$. Unless we need to replace $\omega$ by one of its powers, we may assume that $q$ is a prime number. In accordance with proposition 1.4.2, there exists a positive integer number $r$ such that the degree of $\operatorname{irr}(\omega, \mathbb{k})$ is $2^{r}$. We briefly denote

$$
n=2^{r-1}
$$

therefore the degree of $\operatorname{irr}(\omega, \mathbb{k})$ is $2 n$.

If $p=0$, the roots of $\operatorname{irr}(\omega, \mathbb{k})$ are precisely the nontrivial members of the finite cyclic subgroup $\langle\omega\rangle$ of $D^{\times}$and it follows from theorem 1.1 .3 that $\omega$ and $\omega^{-1}$ are conjugate in $D$. If $p$ is positive, then $2 n$ is the multiplicative order of the image of $p$ in $(\mathbb{Z} / q \mathbb{Z})^{\times}$and the roots of $\operatorname{irr}(\omega, \mathbb{k})$ are

$$
\omega, \omega^{p}, \ldots, \omega^{p^{2 n-1}}
$$

Again by virtue of theorem 1.1.3, $\omega$ and $\omega^{p^{n}}$ are conjugate in $D$.

In agreement with the previous paragraph, there exists $\theta$ in $D^{\times}$such that

$$
\omega^{\theta}=\omega^{-1} \text {, and so } \omega^{\theta^{2}}=\omega \text {, if } p=0,
$$

or there exists a positive integer number $n$ such that

$$
\omega^{\theta}=\omega^{p^{n}}, \text { and so } \omega^{\theta^{2}}=\left(\omega^{p^{n}}\right)^{\theta}=\left(\omega^{\theta}\right)^{p^{n}}=\omega^{p^{2 n}}=\omega \text {, if } p>0 .
$$

We set $\widetilde{\omega}=\omega^{-1}$, if $p=0$, or $\widetilde{\omega}=\omega^{p^{n}}$, otherwise. Since $\theta^{2}$ centralizes $\omega$, the identities

$$
\omega \theta=\theta \widetilde{\omega}, \quad \widetilde{\omega} \theta=\theta \omega, \quad \theta^{*} \omega=\widetilde{\omega} \theta^{*} \text { and } \theta^{*} \widetilde{\omega}=\omega \theta^{*}
$$

hold. We set

$$
\mathbf{i}=\omega-\widetilde{\omega} \text { and either } \mathbf{j}=\theta \text {, if } \theta^{*}=\theta \text {, or } \mathbf{j}=\mathbf{i}^{-1}\left(\theta-\theta^{*}\right) \text {, otherwise. }
$$

Whichever is the case, we set $\mathbf{i}^{2}=a$ and $\mathbf{j}^{2}=b$. It thus follows that $\mathbf{i}^{*}=\mathbf{i}, \mathbf{j}^{*}=\mathbf{j}$ and $\mathbf{j} \mathbf{i}=-\mathbf{i} \mathbf{j}$. It is readily seen that $c=\omega+\widetilde{\omega}$ is symmetric and centralizes both $\mathbf{i}$ and $\mathbf{j}$, therefore the subring $\mathbb{H}$ of $D$ generated by $\mathbf{i}$ and $\mathbf{j}$ over $\mathbb{k}(a, b, c)$ is a division quaternion algebra in which $*$ acts as an orthogonal involution. The normal subgroup $N_{0}=N \cap \mathbb{H}$ of $\mathbb{H}^{\times}$is non central, for

$$
\omega=\frac{1}{2}(\mathbf{i}+c)
$$

belongs to $N_{0}$, but not to $\mathbb{k}(a, b, c)$. In light of theorem $3.2 .3, N_{0}$ and hence $N$ contains a free symmetric pair.

As we have discussed before introducing theorem 3.2.7, if it is intertwined with theorem 3.1.1, one directly concludes the following 
Corollary 3.2.8. Let $D$ be a noncommutative division ring of finite dimension over its center $\mathbb{k}$, a field whose characteristic $p$ is distinct from 2 . Let $*$ be a $\mathbb{k}$-involution in $D$ and $N$ be a normal subgroup of $D^{\times}$. If the symmetric members of $N$ have finite multiplicative order, then $N$ necessarily is central.

Although we lack a proof for the analogous to theorem 3.2.2 without the assumption on the cardinality of the center $k$ of the division ring, the corollary 3.2 .8 maintains the motivation to seek an affirmative answer whenever $\mathbb{k}$ is countable. Inspired by ([3], theorem 1), we leave as a conjecture that the corollary 3.2 .8 remains true if we assume that each symmetric element of $N$ has finite multiplicative order over $k$.

Let $N$ be a non central normal subgroup of the division ring $D$. We let $N$ and $D$ be as in the statement of theorem 3.2.7. In essence, we guaranteed the existence of an element $\theta$ in $D^{\times}$whose square centralizes the member $\omega$ of $N$ of finite multiplicative order. It is possible to abandon both the assumptions on the dimension of $D$ and on the kind of $*$ so that the result remains true whenever $\mathrm{k}$ has characteristic zero or, if it has positive characteristic, the degree of $\operatorname{irr}(\omega, \mathbb{k})$ is even. We register this version below. Its proof is carried out similarly as in theorem 3.2 .7 , observing that we do not need to use proposition 1.4 .2 anymore and carefully replacing $\mathbb{k}$ by $\mathbb{k}_{*}$.

Theorem 3.2.9. Let $D$ be a noncommutative division ring with center $\mathbb{k}$, a field whose characteristic $p$ is distinct from 2. Let $*$ be an involution in $D$ and $N$ be a non central normal subgroup of $D^{\times}$. We assume that $N$ contains a non central symmetric element $\omega$ of finite multiplicative order. If either (i) $p=0$; or (ii) $p>0$ and the degree of $\operatorname{irr}(\omega, \mathrm{k})$ is even; then $N$ contains a free symmetric pair.

Theorem 3.2.9 invites comparison with ([16], theorem 1.2), in which it is assumed that $N$ contains a nonabelian subgroup of odd order, whereas the subgroup we assumed to be contained in $N$ above is of course abelian.

As it was aforementioned, the result concerning free symmetric pairs provided by lemma 3.2.1 may be sharpened, as we testify in the following result, which closes this section.

Theorem 3.2.10. Let $D$ be a division ring with center $\mathrm{k}$, a field whose characteristic is distinct from 2. Let $*$ be an involution in $D$ and $N$ be a non central normal subgroup of $D^{\times}$. If $N$ contains a non central symmetric element $\omega$ such that $[\mathbb{k}(\omega): \mathbb{k}]=2$, then $N$ contains a free symmetric pair. 
Proof: We set $\omega_{1}=\omega$. Regarding $\operatorname{irr}(\omega, \mathrm{k})$ as a member of the polynomial ring $D[X]$, it is divisible by $X-\omega_{1}$, hence there exists $\omega_{2}$ in $D$ such that

$$
\begin{aligned}
\operatorname{irr}(\omega, \mathbb{k}) & =\left(X-\omega_{1}\right)\left(X-\omega_{2}\right) \\
& =X^{2}-\left(\omega_{1}+\omega_{2}\right) X+\omega_{1} \omega_{2}
\end{aligned}
$$

whence $\omega_{1}+\omega_{2}$ and $\omega_{1} \omega_{2}$ belong to $k$. In particular, $\omega_{1} \omega_{2}=\omega_{2} \omega_{1}$. Since

$$
\omega^{2}-\left(\omega_{1}+\omega_{2}^{*}\right) \omega+\omega_{1} \omega_{2}^{*}=\left(\omega^{2}-\left(\omega_{1}+\omega_{2}\right) \omega+\omega_{1} \omega_{2}\right)^{*}=0,
$$

we must have

$$
\operatorname{irr}(\omega, \mathbb{k})=X^{2}-\left(\omega_{1}+\omega_{2}^{*}\right) X+\omega_{1} \omega_{2}^{*}
$$

and necessarily $\omega_{2}^{*}=\omega_{2}$. We conclude that $\omega_{1}+\omega_{2}$ and $\omega_{1} \omega_{2}$ are members of $\mathbb{k}_{*}$. In accordance with theorem 1.1 .3 , there exists $\theta$ in $D^{\times}$such that

$$
\theta^{-1} \omega_{1} \theta=\omega_{2}
$$

therefore we also have

$$
\begin{aligned}
\theta^{-1} \omega_{2} \theta & =\theta^{-1}\left(\omega_{1}+\omega_{2}-\omega_{1}\right) \theta \\
& =\theta^{-1}\left(\omega_{1}+\omega_{2}\right) \theta-\theta^{-1} \omega_{1} \theta \\
& =\left(\omega_{1}+\omega_{2}\right)-\omega_{2} \\
& =\omega_{1},
\end{aligned}
$$

which means that $\theta^{2}$ centralizes $\omega_{1}$ and the equations

$$
\omega_{1} \theta=\theta \omega_{2}, \quad \omega_{1} \theta^{*}=\theta^{*} \omega_{2}, \quad \theta \omega_{1}=\omega_{2} \theta \text { and } \theta^{*} \omega_{1}=\omega_{2} \theta^{*}
$$

hold. We set

$$
\mathbf{i}=\omega_{1}-\omega_{2} \text { and either } \mathbf{j}=\theta \text {, if } \theta^{*}=\theta \text {, or } \mathbf{j}=\mathbf{i}^{-1}\left(\theta-\theta^{*}\right) \text {, otherwise. }
$$

Whatsoever, $\mathbf{i}$ and $\mathbf{j}$ are symmetric, $\mathbf{j} \mathbf{i}=-\mathbf{i} \mathbf{j}$ and so the division subring $\mathbb{H}$ of $D$ generated by $\mathbf{i}$ and $\mathbf{j}$ over $\mathrm{k}$ is a quaternion algebra which is invariant under $*$. We observe that, since $\omega_{1}+\omega_{2}$ belongs to $\mathbb{k}$,

$$
\omega=\frac{1}{2}\left(\mathbf{i}+\left(\omega_{1}+\omega_{2}\right)\right)
$$

is a non central symmetric element of the normal subgroup $N_{0}=N \cap \mathbb{H}$ of $\mathbb{H}^{\times}$. With the aid of the item (i) of theorem 3.2.3. we conclude that $N_{0}$ and hence $N$ contains a free symmetric pair. 


\subsection{A note on characteristic 2}

We propose to illustrate the inconvenience of seeking free symmetric pairs in normal subgroups of division rings whose centers have characteristic 2 . As we have commented before, it is possible to define quaternion $\mathbb{k}$-algebras if $\mathbb{k}$ has characteristic 2. We give an example.

Let $a$ and $b$ be algebraically independent elements over the field $\mathbb{F}_{2}$ with exactly two elements. We denote $\mathbb{k}=\mathbb{F}_{2}(a, b)$ and consider the $\mathbb{k}$-algebra $\mathbb{H}$ generated by elements $\mathbf{i}$ and $\mathbf{j}$, subjected to the relations

$$
\mathbf{i}^{2}+\mathbf{i}+a=0, \quad \mathbf{j}^{2}=b \text { and } \mathbf{j} \mathbf{i}=(\mathbf{i}+1) \mathbf{j} .
$$

These relations between $\mathbf{i}$ and $\mathbf{j}$ are precisely what we require to call $\mathbb{H}$ a quaternion algebra.

A close examination reveals that, since $a$ and $b$ are transcendental over $\mathbb{F}_{2}$, the polynomial

$$
X^{2}+X+a
$$

is irreducible in $\mathbb{k}[X]$, with roots $\mathbf{i}$ and $\mathbf{i}+1$, and

$$
T^{2}+b
$$

is a central irreducible member of the skew-polynomial ring

$$
H=\mathbb{k}(\mathbf{i})[T ; \sigma],
$$

where $\sigma$ denotes the $\mathbb{k}$-automorphism of $\mathbb{k}(\mathbf{i})$ which maps $\mathbf{i}$ to $\mathbf{i}+1$. It follows that we may identify $\mathbb{H}$ with the quotient of $H$ by the ideal

$$
\left(T^{2}+b\right) H
$$

which leads us to conclude that $\mathbb{H}$ is a division ring of dimension 4 over its center $\mathbb{k}$.

Routinely computation reveals that $\{1, \mathbf{i}, \mathbf{j}, \mathbf{k}\}$, where $\mathbf{k}$ denotes the product $\mathbf{i} \mathbf{j}$, is a basis of $\mathbb{H}$ over $\mathbb{k}$, and so we consider the $\mathbb{k}$-linear map $*$ of $\mathbb{H}$ onto itself determined by the conditions

$$
1^{*}=1, \quad \mathbf{i}^{*}=\mathbf{i}+1, \quad \mathbf{j}^{*}=\mathbf{j} \text { and } \mathbf{k}^{*}=\mathbf{k}
$$

It is straightforward to verify that $*$ is an involution in $\mathbb{H}$ which fixes $\mathrm{k}$ elementwise. If

$$
\omega=\alpha+\beta \mathbf{i}+\gamma \mathbf{j}+\delta \mathbf{k}
$$


is a symmetric member of $\mathbb{H}$ with respect to $*$, then $\beta$ necessarily is zero. Regarding the structure of $\mathbb{H}$ as a left $\mathbb{k}(\mathbf{i})$-vector space with basis $\{1, \mathbf{j}\}$, we denote by $P$ the matrix obtained from $\omega$ by right regular representation, explicitly given by

$$
P=\left[\begin{array}{cc}
\alpha & \gamma+\delta \mathbf{i} \\
b(\gamma+\delta(1+\mathbf{i})) & \alpha
\end{array}\right] .
$$

Since $\mathbb{k}$ has characteristic $2, P$ has trace zero, therefore $P^{2}$ is central in $\mathrm{GL}_{2}(\mathbb{k}(\mathbf{i}))$ and $\omega$ is accordingly central in $\mathbb{H}$. We conclude, in opposition to the item (i) of the theorem 3.2.3, that no free subgroup of $\mathbb{H}^{\times}$contains symmetric elements. 



\section{Bibliography}

[1] Amitsur, S. A. Rational identities and applications to algebra and geometry. J. Algebra, 3, 304-359, 1966.

[2] Beidar, K. I., Martindale III, W. S. and Mikhalev, A. V. Rings with generalized identities. Marcel Dekker, New York, 1996.

[3] Chacron, M. A generalization of a theorem of Kaplansky and rings with involution. Michigan Math. J., 20, 45-54, 1973.

[4] Chiba, K. Free subgroups and free subsemigroups of division rings. J. Algebra, 184, 570-574, 1996.

[5] Cohn, P. M. Skew fields. Theory of general division rings. Cambridge University Press, Cambridge, 2008.

[6] Cox, David A. Galois theory. John Wiley \& Son, Hoboken, New Jersey, 2012.

[7] Dieudonné, J. On the structure of unitary groups. Transactions AMS, 87, 367-385, 1958.

[8] Ferreira, V. O. and Gonçalves, J. Z. Free symmetric and unitary pairs in division rings infinite-dimensional over their centers. Israel J. Math., 210, 297-321, 2015.

[9] Ferreira, V. O., Gonçalves, J. Z. and Mandel, A. Free symmetric and unitary pairs in division rings with involution. Int. J. Algebra Comput., 15, 15-36, 2005 .

[10] Ferreira, V., Gonçalves, J. Z. and Sánchez, J. Free Symmetric and Unitary Pairs in the Field of Fractions of Torsion-Free Nilpotent Group Algebras. Algebr. Represent. Theor., 23, 605-619, 2020.

[11] Gonçalves, J. Z. Free groups in subnormal subgroups and the residual nilpotence of the group of units of group rings. Canad. Math. Bull., 27, 365-370, 1984. 
[12] - Free pairs of symmetric and unitary units in normal subgroups of a division rings. J. Algebra Appl., 16, 1750108, 17, 2017.

[13] Gonçalves, J. Z. and Mandel, A. Are there free groups in division rings? Israel J. Math., 53, 69-80, 1986.

[14] Gonçalves, J. Z. and Passman, D. S. Explicit free groups in division rings. Proceedings AMS, 143, 459-468, 2015.

[15] - Free groups in normal subgroups of the multiplicative group of a division ring. J. Algebra, 440, 128-144, 2015.

[16] - Free pairs of symmetric elements in normal subgroups of division rings. J. Algebra, 550, 154-185, 2020.

[17] Gonçalves, J. Z. and Shirvani, M. Free products arising from elements of finite order in simple rings. Proceedings AMS, 133, 1917-1923, 2005.

[18] - Free symmetric and unitary pairs in central simple algebras with involution. Contemp. Math., 420, 121-139, 2006.

[19] - Free groups in central simple algebras without Tits' theorem. Comm. Algebra, 36, 3113-3121, 2008.

$[20]$ - A survey on free objects in division rings and in division rings with an involution. Comm. Algebra, 40, 1704-1723, 2012.

[21] Gonçalves, J. Z., Mandel, A. and Shirvani, M. Free products in algebras I. Quaternion algebras. J. Algebra, 214, 301-316, 1999.

[22] - Free products of units in algebras II. Crossed products. J. Algebra, 233, 567-593, 2000.

[23] Herstein, I. N. Noncommutative rings. Carus Monograph 15. Math. Assoc. Amer., Buffalo, N.Y., 1968.

[24] - Multiplicative commutators in division rings. Israel J. Math., 31, 180-188, 1978.

[25] - Multiplicative commutators in division rings II. Rendiconti del Circolo Matematico di Palermo, 29, 485-489, 1980.

[26] Herstein, I. N. and Montgomery, Susan. A note on division rings with involutions. Michigan Math. J., 18, 75-79, 1971. 
[27] Hua, L.-K. On the multiplicative group of a sfield. Acad. Sinica Sci. Record, 3, 1-6, 1950.

[28] Jacobson, N. Structure theory of algebraic algebras of bounded degree. Annals Math., 46, 695-707, 1945.

[29] - Structure of rings. AMS Colloquium Publication 37, 1964.

[30] - Lectures in abstract algebra II. Linear algebra. Springer-Verlag, New York, 1975.

[31] . Finite-dimensional division algebras over fields. Springer-Verlag, Berlin Heidelberg, 1996.

[32] Janusz, G. Algebraic number fields. Academic Press, New York, 1973.

[33] Kaplansky, I. A theorem on division rings. Canadian J. Math., 3, 290-292, 1951.

[34] . Fields and rings. University of Chicago Press, Chicago, 1972.

[35] Lam, T. Y. A First course in noncommutative rings. Springer, New York, 2013.

[36] Lichtman, A. I. On subgroups of the multiplicative group of skew fields. Proceedings AMS, 63, 15-16, 1977.

[37] - Free subgroups of normal subgroups of the multiplicative group of skew fields. Proceedings AMS, 71, 174-178, 1978.

[38] Nagata, M., Nakayama, T. and Tuzuku, T. On an existence lemma in valuation theory. Nagoya Math. J., 6, 59-61, 1953.

[39] Passman, D. S. The algebraic structure of group rings. Wiley-Interscience, New York, 1977.

[40] Reichstein, Z. and Vonessen, N. Free subgroups of division algebras. Comm. Algebra, 23, 2181-2185, 1995.

[41] Ribenboim, P. The theory of classical valuations. Springer, New York, 2012.

[42] Rotman, J. An introduction to the theory of groups. Springer, New York, 1999.

[43] Scott, W. R. On the multiplicative group of a division ring. Proceedings AMS, 8, 303-305, 1957. 
[44] Tits, J. Free subgroups in linear groups. J. Algebra, 20, 250-270, 1972.

[45] Wedderburn, J. H. M. A theorem on finite algebras. Transactions AMS, 6, 349-352, 1905.

[46] Zalesskii, A. E. Solvable groups and crossed products. Mat. Sb., 67, 154-160, 1965. 


\section{Index}

"denominator", 3

"numerator", 3

Cartan-Brauer-Hua theorem, 2

degree, 23

Dickson's theorem, 2

division quaternion algebra, 6

element

central, 1

skew-symmetric, 5

symmetric, 5

unitary, 5

evaluation map, 3

free pair, 14

free symmetric pair, 23

free unitary pair, 23

involution, 5

of the first kind, 5

of the second kind, 5

of type 1,6

of type II, 6

orthogonal, 7

symplectic, 7

multiplicative group, 1

non absolute field, 7

non archimedean valuation, 13

Ore condition, 3 quaternion

algebra, 6

generators, 6

Scott's theorem, 2

Skolem-Noether theorem, 1

unit, 1

Wedderburn's little theorem, 1 